\title{
Prediction of Global Extinction Conditions and Dynamics in Swirling Non-premixed Flames Using LES/CMC Modelling
}

\author{
Huangwei Zhang ${ }^{1}$ Epaminondas Mastorakos ${ }^{1}$
}

Received: 13 October 2015 / Accepted: 20 November 2015 / Published online: 14 December 2015

(C) The Author(s) 2015. This article is published with open access at Springerlink.com

\begin{abstract}
The Large Eddy Simulation (LES)/three-dimensional Conditional Moment Closure (CMC) model with detailed chemistry is applied to predict the operating condition and dynamics of complete extinction (blow-off) in swirling non-premixed methane flames. Using model constants previously selected to provide relatively accurate predictions of the degree of local extinction in the piloted jet flames Sandia D-F, the error in the blow-off air velocity predicted by LES/3D-CMC in short, recirculating flames with strong swirl for a range of fuel flow rates is within $25 \%$ of the experimental value, which is considered a new and promising result for combustion LES that has not been applied before for the prediction of the whole blow-off curve in complex geometries. The results also show that during the blow-off transient, the total heat release gradually decreases over a duration that agrees well with experiment. The evolution of localized extinction, reactive scalars and scalar dissipation rate is analyzed. It has been observed that a consistent symptom for flames approaching blow-off is the appearance of high-frequency and high-magnitude fluctuations of the conditionally filtered stoichiometric scalar dissipation rate, resulting in an increased fraction of local extinction over the stoichiometric mixture fraction iso-surfaces. It is also shown that the blow-off time changes with the different blow-off conditions.
\end{abstract}

Keywords Global extinction · Blow-off curve · Swirling non-premixed flames · Large eddy simulation · Conditional moment closure

Huangwei Zhang

hz283@cam.ac.uk

Epaminondas Mastorakos

em257@eng.cam.ac.uk

1 Hopkinson Laboratory, Department of Engineering, University of Cambridge,

Cambridge CB2 1PZ, UK 


\section{Introduction}

Flame extinction is a fundamental topic in combustion theory and is also practically important for designing combustion systems [1,2]. It is linked to energy conversion efficiency, pollutant formation and flame suppression in fire safety, etc. Extinction related properties are also significant indices that should be examined for new fuels. Physically, extinction can occur due to removal of critical amount of heat, or chain-branching or chain-carrying radicals from the flame. In turbulent non-premixed combustion, the non-linear coupling between turbulence and chemistry strongly influences the occurrence of localized and global extinction.

Experimental studies on turbulence-chemistry interaction manifested by local extinction and re-ignition have been conducted with single point Raman-Rayleigh-LIF measurements, based on different flame configurations, including simple jet flames [3], piloted jet flames [4], and bluff body flames [5], to name just a few. With the high-speed PLIF and chemiluminescence technique applied for imaging reactive radicals two-dimensionally, it is possible to analyze transient and phenomenological characteristics of local extinction in turbulent non-premixed flames [6-10]. In particular, the turbulent non-premixed combustion in swirling flow fields also attracts lots of attention because of its significant practical applications, such as in gas turbine combustors [11-15]. The turbulence-chemistry interaction in unconfined swirl non-premixed flames with a range of swirl numbers was reviewed in Ref. [16] and it was concluded that the localized extinction may be induced by high shear stress.

Here global extinction is termed for the complete disappearance of the flame because of variations of the flow rates of fuel and/or oxidizer streams in non-premixed combustion systems and is interchangeable with blow-out or blow-off in this study. The blow-out dynamics in turbulent non-premixed jet flames have been investigated theoretically and experimentally, such as their transient behaviors and the intrinsic mechanisms [17, 18]. The estimated blow-out correlations, parameterized by critical flame base locations and/or mixture flow rates, were proposed in Refs. [17-19] based on the simplified theoretical models for lift-off of turbulent non-premixed jet flames [20]. The blow-out limits (i.e. air versus fuel bulk velocities) at both fuel rich and lean compositions in a swirl burner from the University of Michigan (without appreciable bluff body effects) were measured and it is found that the operating conditions for stable swirling flames were extended relative to the nonswirl counterparts, although the extent depends on the swirl number [21, 22]. The results also showed that the compact and strongly recirculating flames, instead of lift-off, can only been observed close to lean blow-out [21]. Different extinction modes, i.e. base and neck blow-off, have been observed in an unconfined swirl burner and the measured stability limits and flame topologies were strongly influenced by the swirl and Reynolds numbers [23]. The blow-off transient, visualized by $\mathrm{OH}^{*}$ chemiluminescence and $\mathrm{OH}-\mathrm{PLIF}$ for confined swirling non-premixed flames, were studied by Cavaliere et al. [15]. The results demonstrate that the flame gradually shrinks in the upstream part of Recirculation Zone (RZ) towards the bluff body surface and can survive in the RZ for a long duration prior to blow-off. The blow-off duration was also quantified. Blow-off dynamics in a dual swirl combustor were related to the helical flame zone and the movement of the flame root [14] and it was concluded that the blow-out starts when the extinction state at the flame root lasts for a time exceeding a critical duration. Furthermore, re-ignition at the flame root was inhibited, which differs from what was observed by Cavaliere et al. [15], in which the re-burning near the bluff body can be seen even at the last stage of blow-off transients. 
Accurately predicting extinction dynamics in turbulent flames is an important measure of the performance of turbulent combustion models in tackling strong turbulence-chemistry interaction. So far, different levels of success in local extinction predictions have been achieved using different combustion models and relevant sub-models. For example, the Flamelet/Progress Variable (FPV) model was proposed to include the flame dynamics effect into the steady flamelet model [24] and applied to capture the extinctions and re-ignition in the Sandia flames D and E [25]. The results show that the degree of localized extinction and re-ignition was successfully predicted and models for the Probability Density Function (PDF) of reaction progress parameter show an important influence on the accurate prediction of the above phenomena. The joint velocity-composition-turbulence frequency PDF model and Filtered probability Density Function (FDF)/stochastic field method were shown to be capable of simulating localized extinction and re-ignition in Sandia flames D-F with good accuracy [26, 27]. The effects of mixing model on extinction prediction were assessed in both studies and it was found that in Reynolds Averaged Navier-Stokes (RANS) simulations the flame structures are more sensitive to the mixing model constant than in Large Eddy Simulations (LES). The Multiple Mapping Conditioning (MMC) model also demonstrates the ability in predicting the correct level of local extinction in Sandia flame series with the reasonable specifications of the respective mixing model constants [28-30]. Concerning the Conditional Moment Closure (CMC) model, its second-order and doubly conditioned variants with simple chemistry were developed to accurately capture the local extinction [31-33], but the application of simple chemical kinetics limits the analysis of local extinction. More recently, the first order CMC model with a detailed mechanism was used in LES to predict local extinction in Sandia flame F [34] and in swirl non-premixed methane flames $[35,36]$. The localized extinction temporal evolution and spatial distribution, and species mass fraction in these different flame configurations were reasonably well calculated, suggesting that the sub-grid scale first-order CMC model that does not include the conditional fluctuations is adequate for extinction when implemented in LES.

Compared to the above computational efforts for local extinction, global extinction simulations are limited. Global extinction is a transient process in which the localized extinction area on the flame front gradually expands until complete extinction occurs. It has been shown recently that the blow-off transient is long relative to estimates of the residence time in the combustor and that empirical correlations of the blow-off condition do not give very accurate results [15, 37-39]. Therefore, in the context of turbulent combustion modeling, the following question can be posed: does a combustion model that can predict reasonably well the degree of local extinction also possesses the ability to predict the global extinction conditions and dynamics in a flame in a realistic geometry? The blow-off dynamics of a swirl-stabilized $n$-heptane spray flame using LES/CMC were investigated and the time evolution of heat release as well as the instantaneous flame behavior were analyzed [40]. Also, for one condition, the simulation predicted an extinguished flame at the experimentallydetermined blow-off velocity. However, the blow-off curve representing a wide range of operating conditions has not been studied systematically in that or other combustion modelling work. The ability to predict, from first principles, a wide range of global blow-off operating points would be of huge importance for the reliability of combustion CFD.

In this study, LES with the sub-grid CMC model is applied to predict blow-off phenomena in confined swirl-stabilized non-premixed methane flames. The following aspects aim to be investigated: (i) ability and accuracy of the CMC model in predicting blow-off conditions, (ii) blow-off dynamics (e.g. evolutions of localized extinction, reactive scalars and scalar dissipation during the global extinction transients) and (iii) blow-off duration. The 
model, flame information and numerical implementations are presented in the next Section. The CMC model and the sub-models are identical to what was used previously to investigate the localized extinction in Sandia flame F [34], and hence the present studies provide an objective assessment whether combustion CFD validated against simple flames with local extinction can be used to predict global blow-off in swirl-stabilized ones. The main findings and discussion are given in Section 3, followed by the conclusions in the final Section 4.

\section{Mathematical Formulation and Flow Considered}

\subsection{LES and CMC models}

The LES governing equations for filtered continuity, momentum and mixture fraction can be derived through filtering the respective instantaneous equations [1]. The anisotropic part of the sub-grid stress tensor is closed with the constant Smagorinsky model and the sub-grid scale viscosity is calculated as [41]

$$
\mu_{\text {sgs }}=c_{k} \bar{\rho} \Delta k^{1 / 2} \text {. }
$$

In Eq. $1, \bar{\rho}$ is the filtered density and $\Delta$ is the filter width calculated as $\Delta=\sqrt[3]{V_{L E S}} \cdot V_{L E S}$ denotes the volume of LES cells. The model constant $c_{k}=0.02$ is used [41]. The algebraic relation based on the local equilibrium assumption is obtained for the sub-grid scale kinetic energy $k$

$$
\mathbf{B}: \tilde{\mathrm{D}}+\bar{\rho} \varepsilon=0,
$$

where $\mathbf{B}$ is the sub-grid scale stress tensor and $\tilde{D}$ is the strain rate. ":" denotes the double inner product of two tensors. The dissipation rate $\varepsilon$ in Eq. 2 is modelled as

$$
\varepsilon=c_{\varepsilon} \frac{k^{3 / 2}}{\Delta},
$$

with the constant $c_{\varepsilon}=1.048$ [41].

The filtered scalar dissipation rate $\tilde{N}$ is calculated through [42]

$$
\tilde{N}=\tilde{N}_{r e s}+\tilde{N}_{s g s}=\underbrace{D \nabla \tilde{\xi} \cdot \nabla \tilde{\xi}}_{\tilde{N}_{\text {res }}}+\underbrace{\frac{c_{N}}{2} \frac{\mu_{s g s}}{\bar{\rho} \Delta^{2}} \tilde{\xi}^{\prime \prime}}_{\tilde{N}_{s g s}},
$$

where $\tilde{N}_{\text {res }}$ and $\tilde{N}_{s g s}$ are the resolved and sub-grid scale scalar dissipation, respectively. $D$ represents the mass diffusivity and is calculated through $D=\mu / \bar{\rho} S c$ with Schmidt number $S c=1.0 . \mu$ is the molecular dynamic viscosity. Here, the constant $c_{N}=42$ [34] is used to estimate the sub-grid scale scalar dissipation rate $\tilde{N}_{s g s}$, which is calibrated against measurements of scalar dissipation in Sandia flame D. The sensitivity of $c_{N}$ on predicting blow-off in a swirling spray flame was investigated by Tyliszczak et al. [40] and it was found that this value gives more satisfactory results than $c_{N}=2[42,43]$ in terms of global extinction transients. $\tilde{\xi}$ and $\widetilde{\xi}_{\widetilde{\xi}^{\prime \prime}}$ are the filtered mixture fraction and its variance, respectively. In the present investigation, $\tilde{\xi}^{\prime \prime}$ is modelled as $\tilde{\xi^{\prime \prime}}=c_{v} \Delta^{2} \nabla \tilde{\xi} \cdot \nabla \tilde{\xi}$ with the constant $c_{v}=0.1$ [44].

The three-dimensional governing equations for the conditionally filtered mass fractions of $\alpha$-th species, i.e. $Q_{\alpha} \equiv \widetilde{Y_{\alpha} \mid \eta}$, are formulated as [35, 36, 45]

$$
\frac{\partial Q_{\alpha}}{\partial t}+\nabla \cdot\left(\widetilde{\mathbf{U} \mid} \eta Q_{\alpha}\right)=Q_{\alpha} \nabla \cdot \widetilde{\mathbf{U} \mid \eta}+\widetilde{N \mid \eta} \frac{\partial^{2} Q_{\alpha}}{\partial^{2} \eta}+\widetilde{\omega_{\alpha} \mid \eta}+e_{f},
$$


in which $\eta$ is the sample space variable for mixture fraction $\xi$. The physical implications of individual terms in Eq. 5, from Left Hand Side (LHS) to Right Hand Side (RHS), are unsteadiness, convection, dilatation, micromixing, chemistry and sub-grid diffusion, respectively. $\widetilde{\mathbf{U} \mid} \eta, \widetilde{N \mid} \eta$, and $\widetilde{\omega_{\alpha} \mid \eta}$ are the conditionally filtered velocity, scalar dissipation rate, and reaction rates of the $\alpha$-th species, respectively. The assumption $\widetilde{\mathbf{U} \mid \eta} \approx \tilde{\mathrm{U}}$ is adopted here. For $\widetilde{N \mid \eta}$, the Amplitude Mapping Closure (AMC) model [46] is used, i.e.

$$
\widetilde{N \mid \eta}=N_{0} G(\eta)
$$

where $N_{0}$ and $G(\eta)$ are respectively

$$
N_{0}=\frac{\tilde{N}}{\int_{0}^{1} \tilde{P}(\eta) G(\eta) d \eta} \text { and } G(\eta)=\exp \left(-2\left[\operatorname{erf}^{-1}(2 \eta-1)\right]^{2}\right) .
$$

The filtered scalar dissipation rate $\tilde{N}$ in Eq. 7 is calculated with Eq. 4. $\tilde{P}(\eta)$ is the Filtered probability Density Function (FDF) and assumed to have a beta-function shape calculated based on $\tilde{\xi}$ and $\widetilde{\xi}^{\prime \prime}$. The first order CMC model, which neglects the conditional fluctuations, is applied for conditional chemical source terms in Eq. 5 such that

$$
\widetilde{\omega_{\alpha} \mid \eta}=\omega_{\alpha}\left(Q_{1}, \ldots Q_{n}, Q_{T}\right)
$$

where $n$ is the number of species and $Q_{T} \equiv \widetilde{T \mid \eta}$ represents the conditionally filtered temperature. The last term in RHS of Eq. 5 is

$$
e_{f}=-\frac{\nabla \cdot\left[\overline{\rho \mid \eta} \tilde{P}(\eta)\left(\widetilde{\mathbf{U} Y_{\alpha} \mid \eta}-\widetilde{\mathbf{U} \mid \eta} Q_{\alpha}\right)\right]}{\overline{\rho \mid \eta} \tilde{P}(\eta)},
$$

and modelled as $e_{f}=\nabla \cdot\left(D_{t} \nabla Q_{\alpha}\right)$ [47]. $D_{t}$ is the turbulent diffusivity and predicted as $D_{t}=\mu_{t} / \bar{\rho} S c_{t}$ with the turbulent Schmidt number $S c_{t}=0.7 . \overline{\rho \mid \eta}$ is the conditionally filtered density.

The governing equation for the conditionally filtered total enthalpy $Q_{h} \equiv \widetilde{h \mid \eta}$ has the same form as Eq. 5 excluding the conditional chemical source term $\widetilde{\omega_{\alpha} \mid \eta}$. To include the convective heat loss effects from solid walls in the sub-grid CMC model, the following term is introduced into the RHS of $Q_{h}$ equation only for CMC cells adjacent to a wall $[48,49]$

$$
\widetilde{q_{W, \Omega} \mid \eta}=-H\left(Q_{T}-T_{W}\right)
$$

where $\widetilde{q_{W, \Omega} \mid \eta}$ is the conditionally filtered volumetric heat loss and $T_{W}$ is the wall temperature. The heat transfer coefficient in Eq. 10, $H$, is predicted through

$$
H=\frac{\tilde{q}_{W, \Omega}}{\overline{\rho \mid \eta} \int_{0}^{1}\left(Q_{T}-T_{W}\right) \tilde{P}(\eta) d \eta} .
$$

$\tilde{q}_{W, \Omega}$ is the filtered volumetric heat loss calculated through volume averaging of the magnitude of the wall surface heat flux $\tilde{q}_{w, S}$ as $\tilde{q}_{W, \Omega}=\int_{\partial \Omega} \tilde{q}_{w, S} d S / V_{L E S}$ where $\partial \Omega$ denotes the faces of the LES cell $\Omega^{L E S}$. Here the surface heat flux magnitude $\tilde{q}_{w, S}$ is estimated from the LES as

$$
\tilde{q}_{w, S}=-\tilde{q}_{w, S}^{l}-\tilde{q}_{w, S}^{t}=\lambda \nabla_{n} \tilde{T}+\lambda_{t} \nabla_{n} \tilde{T},
$$

in which $\tilde{T}$ is the filtered temperature. The gradient of the filtered temperature $\nabla_{n} \tilde{T}$ is aligned with the wall normal direction. The quantities $\tilde{q}_{w, S}^{l}$ and $\tilde{q}_{w, S}^{t}$ denote the individual heat fluxes from laminar and sub-grid scale heat transfer and the latter has been modelled based on the gradient of filtered temperature. In Eq. 12, $\lambda$ and $\lambda_{t}$ are the laminar and sub-grid scale thermal conductivities and predicted through $\lambda=c_{P} \mu / \operatorname{Pr}$ and 
$\lambda_{t}=c_{P} \mu_{t} / \operatorname{Pr}_{t}$, respectively. $c_{P}$ is the specific heat capacity at constant pressure. The molecular and turbulent Prandtl numbers are assumed to be constant with $\operatorname{Pr}=1.0$ and $\operatorname{Pr}_{t}=0.7$, respectively.

The bi-directional data communications between the fine LES and coarse CMC meshes are illustrated in Fig. 1, which is performed for each time step. In particular, the conditionally filtered scalar dissipation rate in the CMC resolution, $\widetilde{N \mid} \eta^{C M C}$, is estimated through FDF-weighted Favre averaging the conditional scalar dissipation, $\widetilde{N \mid} \eta$, over each LES cell constituting one CMC cell

$$
\widetilde{N \mid}^{C M C}=\mathcal{L}_{F D F}(\widetilde{N \mid \eta}) .
$$

For the CMC cells, $\tilde{\xi}^{C M C}$ and $\widetilde{\xi}^{\prime \prime 2} C M C$ are necessary to calculate the beta-shape FDF. They are estimated as [47]

$$
\begin{gathered}
\tilde{\xi}^{C M C}=\mathcal{L}(\tilde{\xi}), \\
\widetilde{\xi}^{\prime \prime 2} C M C=\mathcal{L}\left(\tilde{\xi}^{2}\right)+\mathcal{L}\left(\widetilde{\xi^{\prime \prime 2}}\right)-\mathcal{L}^{2}(\tilde{\xi}) .
\end{gathered}
$$

Additionally, the filtered volumetric heat loss in the $\mathrm{CMC}$ resolution for calculating the heat transfer coefficient $H$ in Eq. 11 is obtained through

$$
\tilde{q}_{W, \Omega}^{C M C}=\mathcal{L}\left(\tilde{q}_{W, \Omega}\right)
$$

In Eqs. 13, 14a, 14b and 15 the operators $\mathcal{L}_{F D F}(x)$ and $\mathcal{L}(x)$ are

$$
\begin{aligned}
\mathcal{L}_{F D F}(x) & =\frac{\int_{\Omega^{C M C}} \bar{\rho} \tilde{P}(\eta) x d \Omega}{\int_{\Omega^{C M C}} \bar{\rho} \tilde{P}(\eta) d \Omega}, \\
\mathcal{L}(x) & =\frac{\int_{\Omega^{C M C}} \bar{\rho} x d \Omega}{\int_{\Omega^{C M C}} \bar{\rho} d \Omega} .
\end{aligned}
$$

They denote the FDF-weighted Favre averaging and Favre averaging operators, respectively. In addition, the volume flux $\tilde{\mathbf{U}} \cdot \mathbf{S}$ (S is the CMC face area vector) and turbulent diffusivity

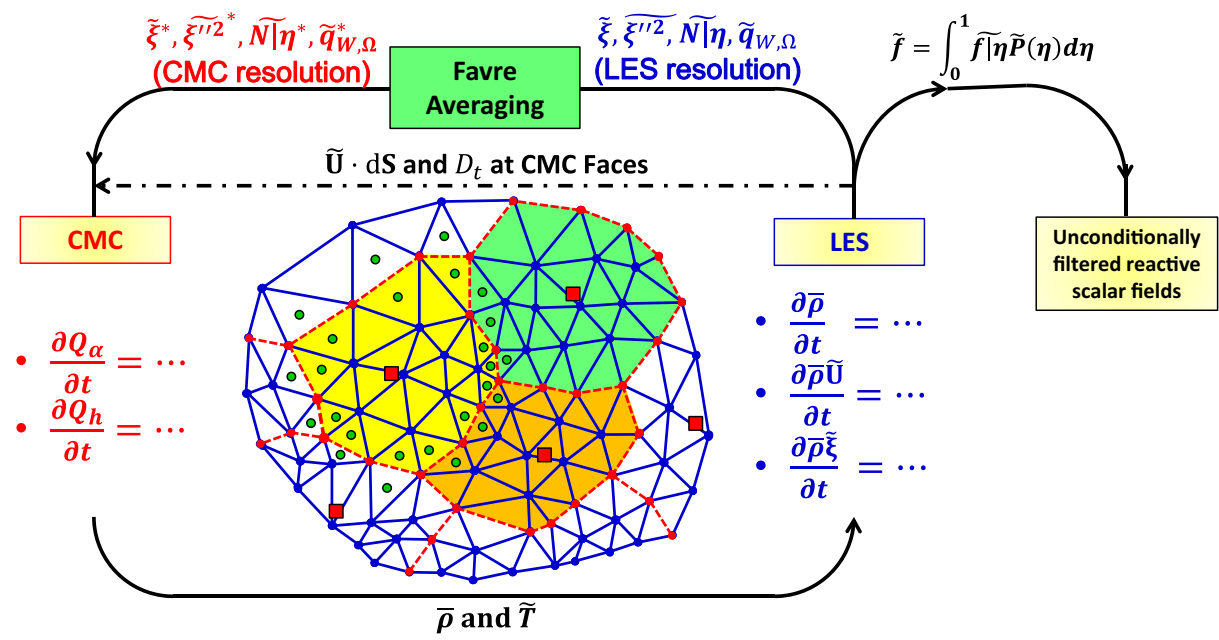

Fig. 1 Schematic of coupling between LES and CMC solvers and CMC cell reconstruction. The twodimensional mesh is the slice through a three-dimensional unstructured LES mesh. Blue lines are LES edges while red lines CMC ones. Green circles represent LES cell centroids while red squares CMC nodes. The cells enclosed by red lines are the reconstructed CMC cell 
$D_{t}$ at the CMC faces are interpolated using the face properties based on the LES results, as shown in Fig. 1. No averaging strategy is introduced for them, which is expected to improve the discretization accuracy for the physical space transport terms in Eq. 5.

The filtered density and temperature, $\bar{\rho}$ and $\tilde{T}$, are provided by CMC to the LES resolution as shown in Fig. 1 . Here $\bar{\rho}$ and $\tilde{T}$, together with the other unconditionally filtered reactive scalar fields (e.g. $\tilde{Y}_{\alpha}$ ) at the LES resolution, are obtained through

$$
\tilde{f}=\int_{0}^{1} \widetilde{f \mid \eta} \tilde{P}(\eta) d \eta
$$

in which $\widetilde{f \mid \eta}$ is the corresponding conditionally filtered scalars provided by the CMC solver.

\subsection{Flow considered and numerical implementations}

Figure 2a shows the burner studied experimentally at the University of Cambridge for localized and global extinction of swirling premixed, non-premixed and spray flames [15]. The dimensions are shown in Fig. 2b. In particular, the size of the rectangular combustor is
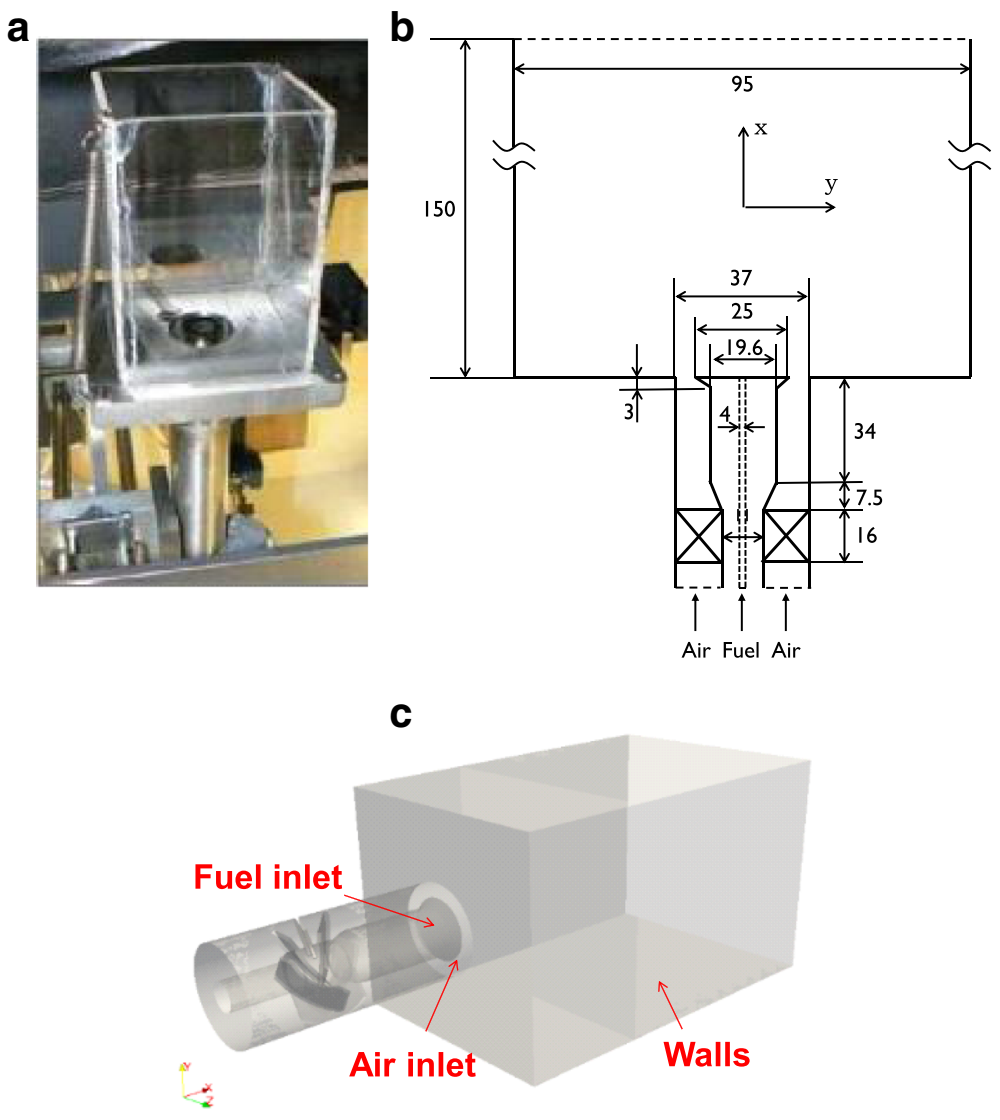

Fig. 2 a Photo and b dimensions (in $\mathrm{mm}$ ) of the burner [52], and $\mathbf{c}$ schematic of computational domain and boundaries 
$0.095 \times 0.095 \times 0.15 \mathrm{~m}^{3}$. A bluff body with diameter $D_{b}=0.025 \mathrm{~m}$ is fitted concentrically in a pipe with $D_{p}=0.037 \mathrm{~m}$ that carries air with swirl imparted by a six-vane swirler. The swirl number $S_{N}$ is calculated following Beer and Chigier's formula [50]

$$
S_{N}=\frac{2}{3} \frac{1-\left(D_{h u b} / D_{s w}\right)^{3}}{1-\left(D_{h u b} / D_{s w}\right)^{2}} \tan \theta,
$$

in which the vane angle $\theta=60^{\circ} . D_{h u b}$ and $D_{s w}$ are the swirler hub diameter and the swirler diameter, respectively and they are $D_{s w}=0.037 \mathrm{~m}$ and $D_{h u b}=0.011 \mathrm{~m}$. Therefore, for the present configuration in Fig. 2a, the swirl number is $S_{N}=1.23$, irrespective of flow conditions. Only the blow-off of non-premixed flames is considered here and the gaseous fuel ( $100 \%$ methane) is injected through a pipe at the center of bluff body top with diameter $D_{f}=0.004 \mathrm{~m}$. The temperature for fuel and air is $298 \mathrm{~K}$.

Mixture fraction space is discretised by 51 nodes, clustered around the stoichiometric mixture fraction $\xi_{s t}=0.055$. In physical space, the Cartesian coordinate origin lies at the center of the circular fuel injector. $x$-axis is the streamwise direction while $y$-and $z$-axis are normal to the lateral combustor walls. Both LES and CMC domains include the rectangular combustor and a downstream hemispherical far-field (not shown in Fig. 2c, but exclude the annulus and the swirler. Around 9 million tetrahedral LES cells are used while the number of the CMC cells is about 140,000. With this LES mesh, over $80 \%$ of the kinetic energy in the chamber can be resolved, based on a mesh sensitivity analysis for the non-reacting flow in this burner [51]. Additionally, the ratio of the LES cell size to Kolmogorov length scale $\eta_{k}$ in the chamber can vary between 15 and 30, in which $\eta_{k}$ is estimated from the typical velocity fluctuation of $20 \%$ of the bulk inlet velocity and a length scale equal to shear layer thickness (about $0.005 \mathrm{~m}$ in this flow) [52]. The CMC cells are arbitrarily polyhedral and are reconstructed through selecting the CMC faces (red lines in the two-dimensional mesh schematic inset of Fig. 1) from the LES cell faces (blue lines) encompassing the CMC nodes (red squares). The algorithms for the CMC mesh re-generation in physical space are detailed in Refs. [45] and [51]. The local refinement of unstructured LES and CMC meshes brings about the flexibility to capture the sharp variations of (i) flow and mixing fields (e.g. the shearing layers resulting from the swirling air and fuel jets) and (ii) conditional reactive scalar fields (e.g. the high scalar dissipation regions). In addition, the near-wall LES cell near the bluff body surface is refined to $y^{+}<4$, to accurately predict the interaction between the flames and bluff body surface resulting from convective heat transfer.

For CMC boundaries in mixture fraction space, $\eta=0$ corresponds to air and $\eta=1$ to fuel, both at $298 \mathrm{~K}$. For LES boundaries presented in Fig. 2c, at all the walls, nonslip condition is applied for the velocities and zero gradient for the mixture fraction. The wall temperature $T_{W}$ was not measured in the experiment but assumed to be $298 \mathrm{~K}$. Zero pressure gradient and Dirichlet velocity and mixture fraction conditions are enforced for the air and fuel inlets which are at the burner exit plane while fixed total atmospheric pressure for the far-field boundaries. About the CMC boundaries in physical space, the inert mixing solutions are specified in the air and fuel inlets as well as at all the walls, while zero gradient extrapolation is enforced at the far-field outlet. All the CMC cells are initialized with the fully burning solutions of $Q_{\alpha}$, which are from stand-alone "OD-CMC"calculations (Eq. 5 without conditional convection, dilatation and sub-grid diffusion terms) with conditional scalar dissipation model parametrized by $N_{0}=501 / \mathrm{s}$ in Eq. 6 .

Both the LES and CMC governing equations are discretized using finite volume method. For LES, an OpenFOAM solver with low Ma number assumption is used. The PISO algorithm is used for the velocity-pressure coupling and a second-order implicit CrankNicholson scheme for time marching. The time step is $\Delta t=2 \times 10^{-6} \mathrm{~s}$ in this work and 
the CFL number in the main flow and flame regions is less than 0.5. For CMC, a newly developed in-house finite volume solver is adopted for solving Eq. 5 and is interfaced with the LES solver. Full operator splitting is used for the terms in Eq. 5. The conditional chemistry terms $\widetilde{\omega_{\alpha} \mid \eta}$ are calculated by the ODE solver VODPK [53] with ARM2 mechanism which includes 19 species and 15 reactions [54]. The micromixing term in Eq. 5 is calculated with TDMA method. Time integration is performed by first-order Euler scheme for the conditional convection, dilatation and sub-grid diffusion terms. The first-order upwind and second-order central differencing schemes are used for discretizing conditional convection and sub-grid diffusion terms, while about the conditional dilatation term the linear interpolation is used for calculating the velocities at the CMC faces. Both LES and CMC solvers are scalable and parallelized with Message Passing Interface (MPI) libraries. Eighty processors are used for the present investigations and approximately $0.002 \mathrm{~s}$ of physical time can be achieved with 24-hour wall clock time.

\subsection{Blow-off simulation strategy}

The flame condition is parameterized by the fuel and air bulk velocities (i.e. $U_{f}$ and $U_{a}$ ) and a few pairs of such values that give rise to global blow-off in the experiment are reproduced in Fig. 3. A large number of extra $\left(U_{f}, U_{a}\right)$ conditions are investigated with LES. Most of these runs are done by starting with the identical instantaneous flow and scalar fields of a stable flame, which follows the procedure in the experiment [15]. In the blow-off experiment the fuel flow rate is fixed and the air flow rate is gradually increased in steps of around $2 \%(0.258 \mathrm{~m} / \mathrm{s})$ every $20 \mathrm{~s}$ until the blow-off is reached in the fuel-lean condition. This procedure is repeated and the blow-off air bulk velocity, $U_{B O \text {,exp }}$, is then obtained through averaging the critical velocities from each experiment for the investigated fuel flow rate. In principle, such operation is also feasible in LES/3D-CMC but in light of the prohibitive

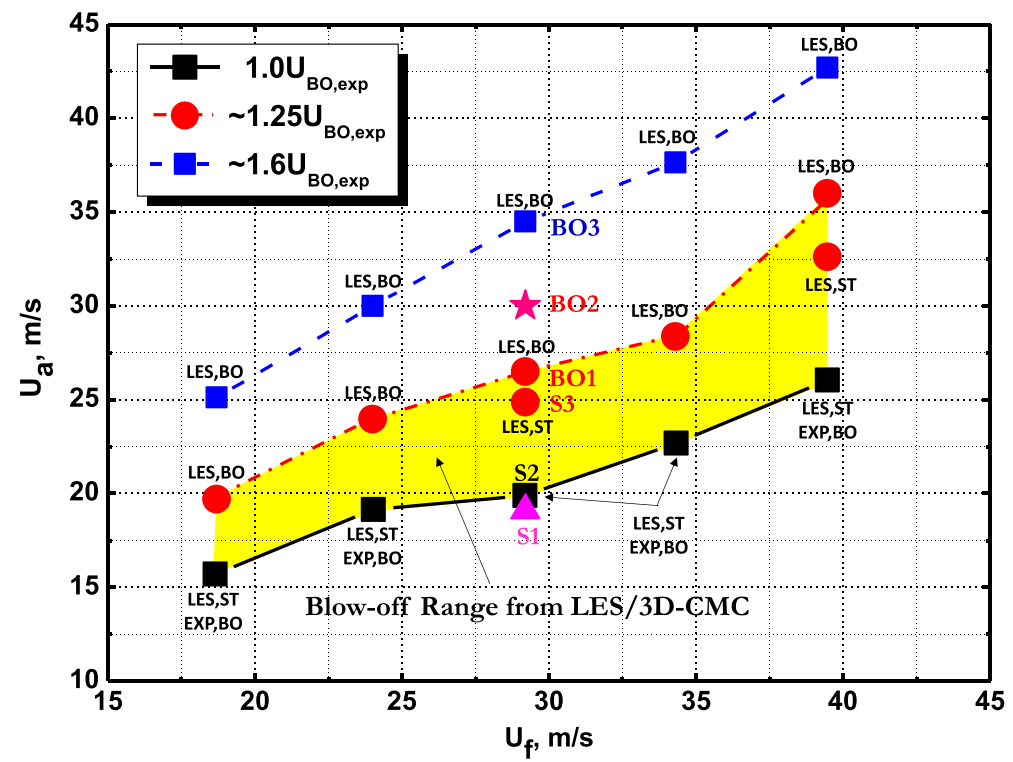

Fig. 3 Air axial bulk velocity at blow-off as a function of the fuel jet velocity from experiments [15] and LES/3D-CMC. LES, BO: blow-off in LES, LES, ST: stable flames in LES, EXP, BO: blow-off in experiments 
computational cost, in this study the air and/or fuel velocities are changed to the required values in one go for each simulation.

To examine the occurrence of blow-off in LES, the quantity of the volume integrated heat release rate $Q$ is calculated from $Q=\int_{V} \tilde{\dot{q}} d V$. Here $V$ represents the whole computational domain. $\tilde{\dot{q}}$ denotes the resolved heat release rate $\tilde{\dot{q}}=\int_{0}^{1} \widetilde{\dot{q} \mid \eta} \tilde{P}(\eta) d \eta$, in which the conditionally resolved heat release rate is predicted as $\widetilde{\dot{q} \mid \eta}=\sum_{\alpha=1}^{n} W_{\alpha} h_{\alpha} \widetilde{\omega_{\alpha} \mid \eta} . W_{\alpha}$ and $h_{\alpha}$ are the molecular weight and specific enthalpy of the $\alpha$-th species, respectively. In this investigation, the blow-off event is assumed to occur when the integrated heat release rate $Q$ reaches below $1 \%$ of its initial value that is integrated from the initial field. Accordingly, the duration within which $Q$ evolves from the initial to low $(<1 \%)$ values is defined as the blow-off time $\tau_{B O}$. If in the whole simulated time (typically $0.05-0.07 \mathrm{~s}$ ), $Q$ flutuates with respect to some stable value, then no blow-off happens under this operating condition and the flame is globally stable. In the measurements [15], the quantification of the individual blow-off transients is based on evolutions of the corresponding area intergation $\mathrm{OH}^{*}$ signals taken from the fast imaging of $5 \mathrm{kHz} \mathrm{OH} *$ chemiluminescnece. The reported experimental blow-off time $\tau_{B O}$ is extracted from the averaged time series of area intergated $\mathrm{OH}^{*}$ emissions compiled from repeated experiments for the specific operating conditions. The experimental $\tau_{B O}$ is defined as the time needed for the $\mathrm{OH}^{*}$ emissions to be reduced from $90 \%$ to $10 \%$ of the initial value. Therefore $\tau_{B O}$ from the LES only corresponds to a single event but the experimental one in Ref. [15] is in an averaged sense. Additionally, very low or zero heat liberated from turbulent non-premixed flames can be only viewed as a necessary condition for extinction [35]. As such, in the computations the evolutions of key reaction scalars are examined as well, as a significant supplement to a $Q$-based criterion.

The flames selected for further analysis are listed in Table 1: three (i.e. S1, S2 and S3) are computationally stable, while other three (i.e. BO1, BO2 and BO3) cases result in computational blow-off and hence global extinction. These six cases share the identical fuel bulk velocity (i.e. $U_{f}=29.2 \mathrm{~m} / \mathrm{s}$ ) but have the successively increased air bulk velocity $U_{a}$ from $\mathrm{S} 1$ to BO3. Since the velocity statistics at the chamber air inlet marked in Fig. 2c are not available from experiment for all these conditions, a non-reacting RANS with the S1 operating conditions and complete computational domain (i.e. swirler, annulus, combustion chamber and far-field) presented in Fig. $2 \mathrm{c}$ is first conducted. Excellent agreement with measurements is obtained about the mean and r.m.s. of axial and swirl velocity components [51]. Then for all the above mentioned LES cases and other cases in Fig. 3, the profiles of three velocity components (axial, swirl and radial velocities) at the air inlet are interpolated and scaled from the non-reacting RANS based on the ratio of the target values (e.g. 19.9 $\mathrm{m} / \mathrm{s}$ for $\mathrm{S} 2$ ) to the $\mathrm{S} 1$ air axial bulk velocity, $19.1 \mathrm{~m} / \mathrm{s}$. Thus for all the simulated cases, the swirl number estimated from Eq. 18 is kept constant as $S_{N}=1.23$. Top-hat profiles are used at fuel inlets for all the LES cases in Table 1. White noise with $5 \%$ intensity is applied at both the air and fuel inlets. The effects of inlet turbulence (i.e. white noise and synthetic eddy method) have been studied with LES of the above non-reacting case [51]. It is found that the difference of predicted Reynolds stress is only limited to shear layers between the swirling air and chamber recirculating flows at the upstream locations, i.e. $x<8 \mathrm{~mm}$. Beyond that, the inlet turbulence shows a negligible influence. Simulations of S2, S3, BO1, $\mathrm{BO} 2$ and $\mathrm{BO} 3$ are conducted through being initialized from well-developed LES and CMC fields of the globally stable case $\mathrm{S} 1$. In addition, for $U_{f}=29.2 \mathrm{~m} / \mathrm{s}$, the experimentally critical blow-off case is $\mathrm{S} 2$ and therefore the experimentally-determined blow-off air velocity for $U_{f}=29.2 \mathrm{~m} / \mathrm{s}$ is $U_{B O, \text { exp }}$ is $19.9 \mathrm{~m} / \mathrm{s}$ [15]. The ratio of axial air velocity to $U_{B O \text {,exp }}$, $U_{a} / U_{B O \text {,exp }}$, the global equivalence ratio $\phi_{g}$ and momentum ratio of air and fuel axial velocities, $\rho_{a} U_{a}^{2} / \rho_{f} U_{f}^{2}$, are also tabulated in Table 1 . 
Table 1 Selected information of simulated cases

\begin{tabular}{lllllll}
\hline Cases & $U_{a}(\mathrm{~m} / \mathrm{s})$ & $U_{f}(\mathrm{~m} / \mathrm{s})$ & $\begin{array}{l}U_{a} / U_{B O, \text { exp }} \\
(\%)\end{array}$ & $\phi_{g}$ & $\rho_{a} U_{a}^{2} / \rho_{f} U_{f}^{2}$ & $\begin{array}{l}\text { Blow-off or stable } \\
\text { in LES/3D-CMC }\end{array}$ \\
\hline S1 & 19.1 & 29.2 & 95 & 0.305 & 0.82 & Stable \\
S2 & 19.9 & 29.2 & 100 & 0.293 & 0.88 & Stable \\
S3 & 25.0 & 29.2 & 126 & 0.233 & 1.40 & Stable \\
BO1 & 26.5 & 29.2 & 133 & 0.220 & 1.57 & Blow-off \\
BO2 & 30.0 & 29.2 & 151 & 0.194 & 2.01 & Blow-off \\
BO3 & 34.5 & 29.2 & 173 & 0.169 & 2.66 & Blow-off \\
\hline
\end{tabular}

\section{Results and Discussion}

\subsection{Blow-off curve}

The critical air axial bulk velocity $U_{a}$ at blow-off as a function of the fuel jet velocity $U_{f}$ was measured by Cavaliere et al. [15] and the data are the squares marked by "EXP, BO" in Fig. 3. Above the black dashed curve, there is no stable flame in the experiments (i.e. blow-off occurs) [15]. The six simulated cases in Table 1 are also denoted as S1-S3 and BO1-BO3 in Fig. 3. To obtain the critical air axial bulk velocity, $U_{B O, L E S}$, corresponding to the experimental $U_{f}$ operating conditions, a trial-and-error strategy with LES and CMC modelling is performed. Specifically, S2 and other four cases corresponding to the experimental blow-off curve (i.e. 1.0 $U_{B O}$, exp in Fig. 3) are first simulated and it turns out that computationally severe local extinction is observable but the flames in these cases do not disappear within sufficiently long computational time. Hence, the exact blow-off conditions from the experiments give a severely fragmented (as we will see later) but still ignited flame in the simulations. The curve of $1.0 U_{B O}$, exp is therefore thought of as the lower blow-off boundary to locate $U_{B O}, L E S$ from LES/3D-CMC. Then, BO3 and other four cases (denoted by blue rhombuses in Fig. 3) are run with the air bulk velocities of approximately $1.6 U_{B O}$, exp and the results show that all these five cases result in blow-off (marked by "LES, BO" in Fig. 3) in the computations. This means that LES/3D-CMC reproduces the blow-off condition to an accuracy better than $60 \%$ for a range of fuel velocities $U_{f}$. Further, a similar effort was done to examine the blow-off likelihood by LES/3D-CMC for air velocities exactly $25 \%$ higher than the experimental blow-off velocity. In particular, the cases with $\left(U_{f}, U_{a}\right)=(29.2,25)$ and $(39.5,32.5)$ demonstrate stable flame features (marked by "LES, ST") but possess severe localized extinctions, while the other three with $1.25 U_{B O}$, exp show blow-off (marked by "LES, BO"). Additional simulations show that, for $U_{f}=29.2 \mathrm{~m} / \mathrm{s}$ and $39.5 \mathrm{~m} / \mathrm{s}$, the air bulk velocities that can make the flame undergo global extinction in LES/3D-CMC are $26.5 \mathrm{~m} / \mathrm{s}$ and $36.0 \mathrm{~m} / \mathrm{s}$, respectively. These two points are marked by "LES, BO" in Fig. 3 and are combined with three blow-off points with $U_{a}=1.25 U_{B O}$, exp to constitute the red dashed line (termed as roughly $1.25 U_{B O}$, exp curve hereafter), which is viewed as the upper blow-off boundary from LES/3D-CMC. The trend that increasing the fuel velocity results in a higher air velocity for blow-off is correctly captured. Therefore, the blow-off range in the computations, denoted as the highlighted yellow region in Fig. 3, suggests that the blow-off condition predicted by the model (i.e. $U_{B O}, L E S$ ) lies between the $1.0 U_{B O}$, exp (lower boundary) and $1.25 U_{B O}$, exp. (upper boundary) curves. 
As far as the authors are aware, capturing the global blow-off condition with combustion LES has not been demonstrated yet. This novel result from Fig. 3 demonstrates that the present LES/3D-CMC model, using model constants $c_{N}$ of sub-grid scalar dissipation calibrated against measurements of scalar dissipation in the Sandia flame D [34], which also results in reasonably predictions of the degree of local extinction in Sandia flame $\mathrm{F}$ [34], predicts, for a range of fuel jet velocities, an air blow-off velocity within $25 \%$ of the experimentally-determined value. This suggests that the sub-grid CMC model is reasonably good at predicting the strong turbulence-chemistry interaction and this accuracy is promising and might at present even be considered acceptable from an engineering perspective.

\subsection{Blow-off characterization}

The time records of the volume integrated heat release rate $Q$ for the stable flames ( $\mathrm{S} 1$, $\mathrm{S} 2$ and $\mathrm{S} 3$ ) and blow-off cases (BO1, BO2 and $\mathrm{BO} 3)$ detailed in Table 1 are presented in Fig. 4. As shown in Fig. 4a, although the fuel and/or air inlet velocities are changed to the desired bulk velocities when the simulation starts, the integrated flame heat release rate $Q$ demonstrate the pronounced "resistance" (lasting roughly $0.02 \mathrm{~s}$ for the stable flame cases in Fig. 4a). After $t=0.02 \mathrm{~s}, Q$ of S1, S2 and S3 cases start to evolve differently due to their different $U_{a}$ but individually maintain relatively stable values although some low-frequency fluctuations can be found, independent of $U_{a}$. Their time-averaged $Q$ values compiled from the time records after $t=0.02 \mathrm{~s}$ are $7.09,6.49$ and $3.65 \mathrm{~kW}$, respectively, well below the nominal $12.38 \mathrm{~kW}$, which is estimated by the fuel flow rate times the $\mathrm{CH}_{4}$ heat of combustion in the standard state [2]. This finding, in a global sense, indicates the considerable finite-rate chemistry effects in the investigated swirling flames and also the gradual weakening of flame reactivity with increased air axial bulk velocities from $\mathrm{S} 1\left(U_{a}=\right.$ $19.1 \mathrm{~m} / \mathrm{s})$ to $\mathrm{S} 3\left(U_{a}=25.0 \mathrm{~m} / \mathrm{s}\right)$.

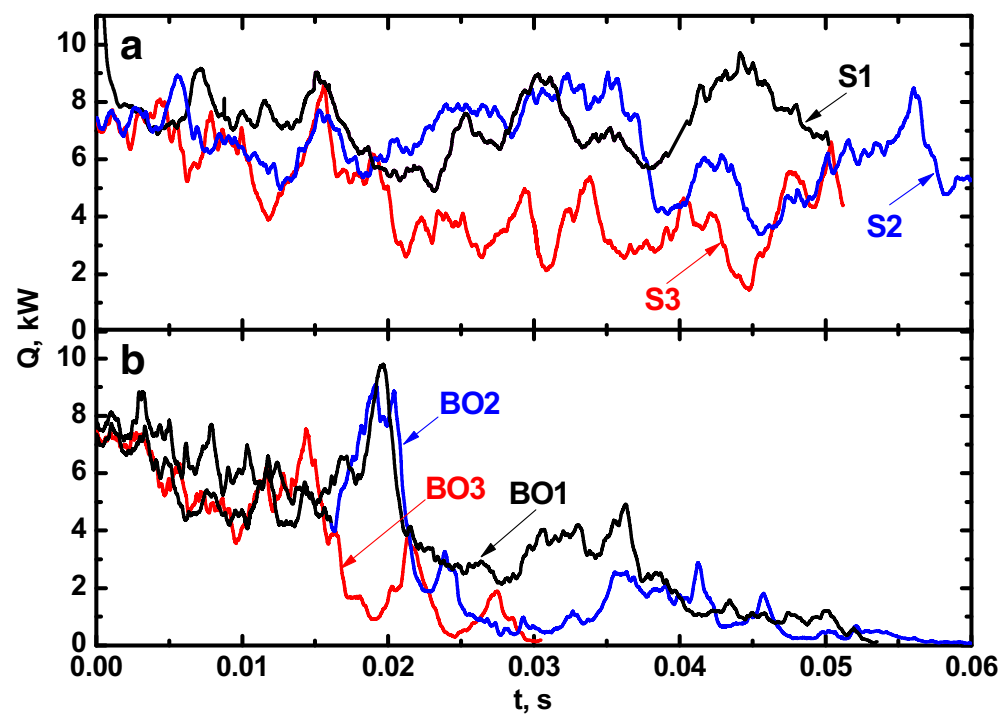

Fig. 4 Time records of total heat release from a stable and $\mathbf{b}$ blow-off cases 
For blow-off case BO1 with $U_{a}=26.5 \mathrm{~m} / \mathrm{s}$, the total heat release rate $Q$ is shown in Fig. 4b: $Q$ gradually decreases, accompanied by the considerable low-frequency fluctuations and also the abrupt increase of $Q$ at $t=0.02 \mathrm{~s}$, and eventually reaches zero, indicating the occurrence of the complete blow-off in the LES. This trend is explicitly different from those of stable cases in Fig. 4a. With increased $U_{a}$ being $30 \mathrm{~m} / \mathrm{s}, \mathrm{BO} 2$ shows the basically similar $Q$ variations to BO1 towards blow-off but faster decay of $Q$ since $t=0.021 \mathrm{~s}$. In BO2, relatively low $Q$ with limited increment, corresponding to very weak combustion with fluctuating heat liberation, persists for a long duration, i.e. between $t=0.03 \mathrm{~s}$ and $0.05 \mathrm{~s}$. The blow-off time obtained from $\mathrm{BO} 1$ and $\mathrm{BO} 2$ is $\tau_{B O} \approx 0.055 \mathrm{~s}$ and $0.057 \mathrm{~s}$, close to the measured critical result of $0.0466 \mathrm{~s}$. One should be reminded of the fact that this measured critical blow-off time of $0.0466 \mathrm{~s}$ is in statistically mean sense and obtained from the experiments with $\mathrm{S} 2$ operating conditions, instead of $\mathrm{BO} 1$ or $\mathrm{BO} 2$ [15]. $\tau_{B O}$ of $\mathrm{BO} 1$ and BO2 was not measured in the experiment. In Fig. $4 \mathrm{~b}$, one can also see that when $U_{a}$ is increased to $34.5 \mathrm{~m} / \mathrm{s}$ in $\mathrm{BO} 3, \tau_{B O}$ is decreased to about $0.03 \mathrm{~s}$, a trend consistent with the observations from premixed flame blow-off experiments [37, 38]. Furthermore, the peaks of $Q$ time series after $t=0.014 \mathrm{~s}$ are intermittently discernible before BO3 extinguishes completely.

The time evolutions of three-dimensional iso-surfaces of filtered heat release rate $(\tilde{\dot{q}}=$ $60 \mathrm{MJ} / \mathrm{m}^{3} \mathrm{~s}$ ) overlaid by the filtered temperature $\tilde{T}$ for S3 and BO2 are presented in Figs. 5 and 6 , respectively. Before $t=0.018 \mathrm{~s}$ in Fig. $5 \mathrm{a}-\mathrm{c}$, heat release regions are extensive and comparatively continuous in the Inner Recirculation Zone (IRZ) near the bluff body and there are also considerable high $\tilde{T}$ areas on these iso-surfaces. Then the heat release regions become small and fragmented as shown in Fig. 5d but iso-surfaces always exist near the

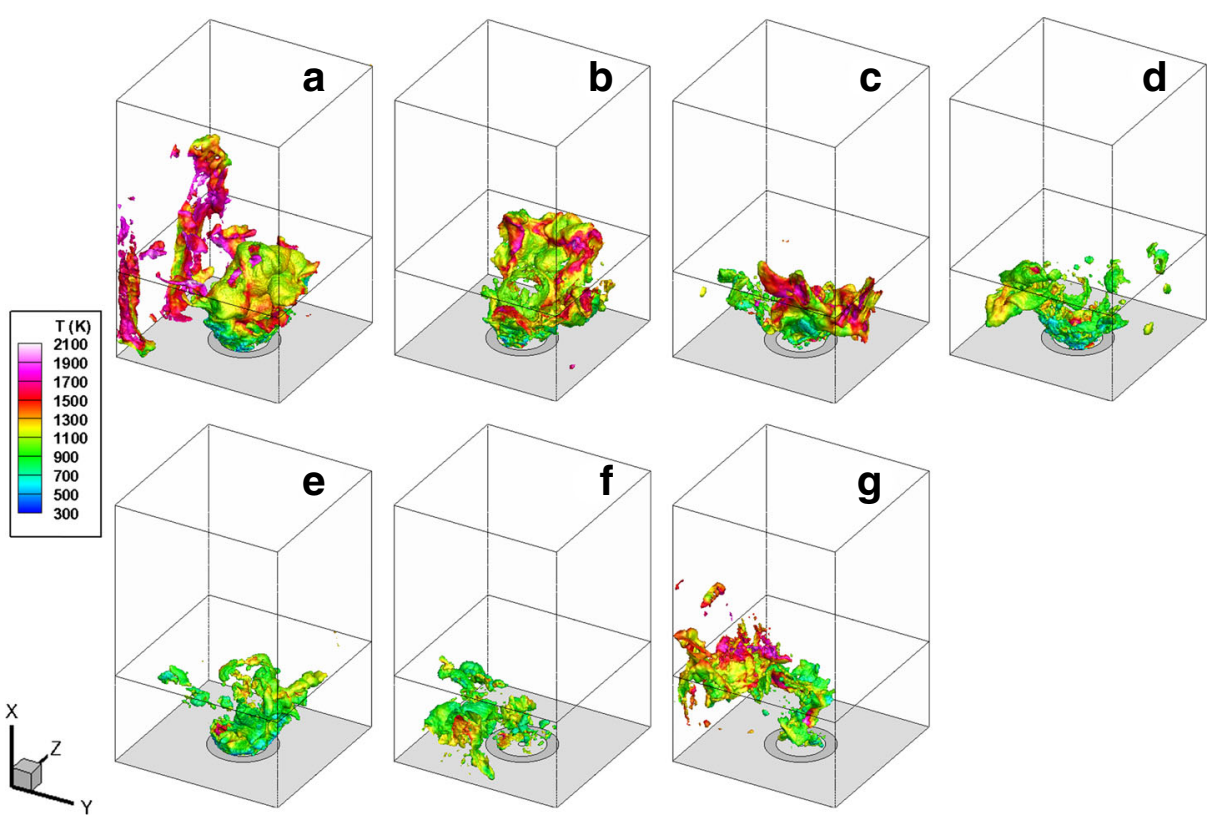

Fig. 5 Time evolution of iso-surfaces of filtered heat release rate $\left(60 \mathrm{MJ} / \mathrm{m}^{3} \mathrm{~s}\right)$ coloured by temperature in $\mathrm{S} 3: \mathbf{a} t=0 \mathrm{~s}, \mathbf{b} t=0.009 \mathrm{~s}, \mathbf{c} t=0.018 \mathrm{~s}, \mathbf{d} t=0.027 \mathrm{~s}, \mathbf{e} t=0.036 \mathrm{~s}, \mathbf{f} t=0.045 \mathrm{~s}$ and $\mathbf{g} t=0.05 \mathrm{~s}$. The box edges denote the chamber size 


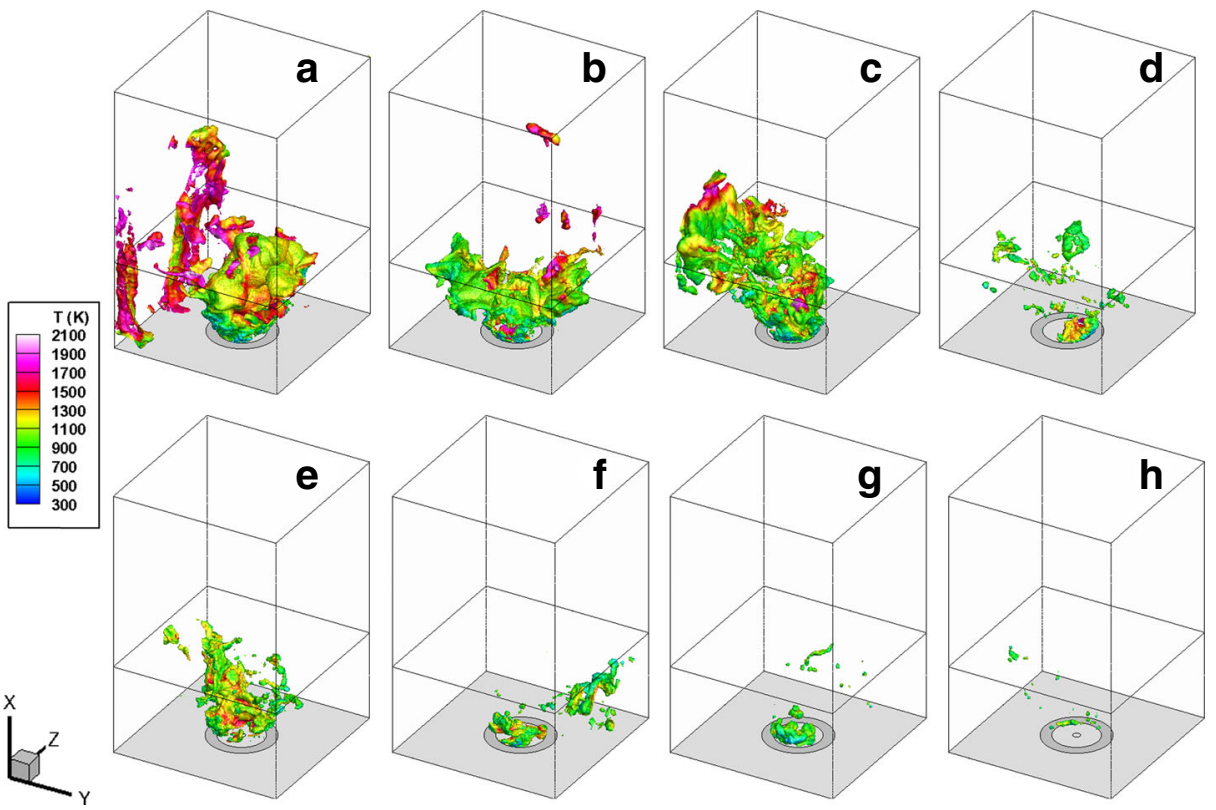

Fig. 6 Time evolution of iso-surfaces of filtered heat release rate $\left(60 \mathrm{MJ} / \mathrm{m}^{3} \mathrm{~s}\right)$ coloured by temperature in $\mathrm{BO} 2: \mathbf{a} t=0 \mathrm{~s}, \mathbf{b} t=0.009 \mathrm{~s}, \mathbf{c} t=0.018 \mathrm{~s}, \mathbf{d} t=0.027 \mathrm{~s}, \mathbf{e} t=0.036 \mathrm{~s}, \mathbf{f} t=0.045 \mathrm{~s}, \mathbf{g} t=0.054 \mathrm{~s}$ and h $t=0.061 \mathrm{~s}$. The box edges denote the chamber size

bluff body until $t=0.05 \mathrm{~s}$ in Fig. $5 \mathrm{~g}$. This is consistent with the time history of S3 total heat release in Fig. 4a. For BO2, similar to the S3 results in Fig. 5a-c, the liberated heat is pervasive in the upstream regions of IRZ presented in Fig. 6a-c. Nevertheless, at $t=0.027$ $\mathrm{s}$ in Fig. 6d, the heat release is greatly reduced although at $t=0.036 \mathrm{~s}$ in Fig. 6e some degree of recovery appears. Then the $\tilde{\dot{q}}$ iso-surfaces gradually diminish again and basically disappear at $t=0.061 \mathrm{~s}$ when $\mathrm{BO} 2$ has extinguished based on discussed in Fig. $6 \mathrm{~b}$.

Two additional observations from Fig. 6 worth noting are: (i) during the $\mathrm{BO} 2$ transient towards complete extinction, finite heat release exists very close to the circular bluff body surface for a long period (see Fig. 6f and g) and (ii) due to the essential solid-body rotation in the IRZ, the heat is intermittently transported from the IRZ toward the lateral walls (see Fig. $6 \mathrm{c}$ and f), where it is accumulated and hence the local $\tilde{\dot{q}}$ increase is expected due to the not too high scalar dissipation there and reverse flows. These two points can respectively justify the features of total heat release rate demonstrated by $\mathrm{BO} 2$ in Fig. $6 \mathrm{~b}$, i.e. the long persistence with low $Q$ and intermittent peaks, which are also seen from the time series of area integrated $\mathrm{OH}^{*}$ emissions from the experiment [15]. Therefore, generally speaking, the current LES/3D-CMC modelling correctly predicts the basic transient behaviors of blow-off in swirling non-premixed flames. Interestingly, the LES/3D-CMC simulations of blow-off of $n$-heptane spray flames in the same burner [40] does not show this intermittent behaviour, which may be attributed to the strong correlations between combustion and evaporation, i.e. weakened reactions leads to cooler recirculation zone and hence weakened evaporation, a process leading to a quicker blow-off event in spray flames compared to the gas-fuelled flames. This discrepancy between non-premixed and spray flames is also indicated by the respective blow-off experiments [15]. 


\subsection{Localized extinction and re-ignition in blow-off}

Three-dimensional iso-surfaces of filtered stoichiometric mixture fraction $\xi_{s t}$ coloured by conditionally filtered $\mathrm{OH}$ mass fraction at stoichiometry $\widehat{Y_{O H} \mid \xi_{s t}}$ from blow-off event BO2 are plotted in Fig. 7a. A large fraction of the iso-surface experiences the extinction (visualized as blue, i.e. low $\widehat{Y_{O H} \mid \xi_{s t}}$ ), although fully reactive regions with high $\widetilde{Y_{O H} \mid \xi_{s t}}$ (red surfaces) still exist. The extinction regions on the iso-surface in Fig. 7a are more extensive and spatially continuous than those in the simulated stable flames like S1 and S2 (results not shown here). To quantify the evolutions of $\xi_{s t}$ iso-surfaces and the local extinction evolutions on them for both stable and blow-off cases, the time sequences of the $\xi_{s t}$ iso-surface area, $\Sigma$, and its extinguished fraction $f_{\Sigma, \text { ext }}$ from $\mathrm{S} 3$ and $\mathrm{BO} 2$ are presented in Fig. $7 \mathrm{~b}$ and c, respectively. Here the extinguished fraction $f_{\Sigma, e x t}$ is calculated as the area ratio of $\xi_{s t}$ iso-surface regions with $\widehat{Y_{O H} \mid \xi_{s t}}<0.00024$ to the total iso-surface $\Sigma$, i.e.

$$
f_{\text {ext }}=\frac{\Sigma \mid\left(\widehat{Y_{O H} \mid \xi_{s t}}<0.00024\right)}{\Sigma},
$$

in which the threshold, i.e. $\widetilde{Y_{O H} \mid \xi_{s t}}<0.00024$, is determined by examining the OH distributions in mixture fraction space during the onset of extinction [35]. Hence $f_{\Sigma \text {, ext }}=1$ means the complete extinction over the entire $\xi_{s t}$ iso-surface, whilst $f_{\Sigma, e x t}=0$ fully burning. It can be seen from Fig. $7 \mathrm{~b}$ that $\Sigma$ of S3 fluctuates around a stable value, around 0.012 $\mathrm{m}^{2}$. This feature is different from the results for the blow-off event BO2 in Fig. 7c, in which case $\Sigma$ shows a slight decrease with some fluctuations. Also, this trend of $\mathrm{BO} 2$ case is contrary to the results from a recent DNS of planar, non-premixed ethylene jet flames [55], where the area of instantaneous $\xi_{s t}$ iso-surfaces increases as the local extinction degree increases. This may be attributed to the different mixing levels in the current recirculating flows and the jet ones, and their response to the occurrence of extinction. The extinguished fraction, $f_{\Sigma, \text { ext }}$, from $\mathrm{BO} 2$ increases from a initially low value to around unity. In addition,

a

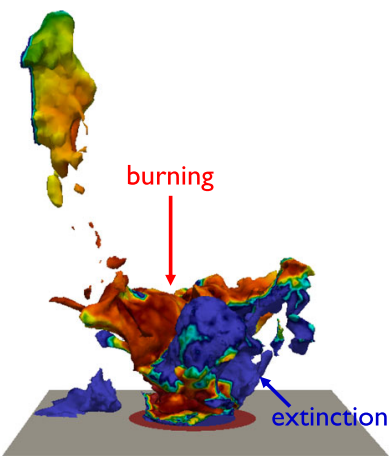

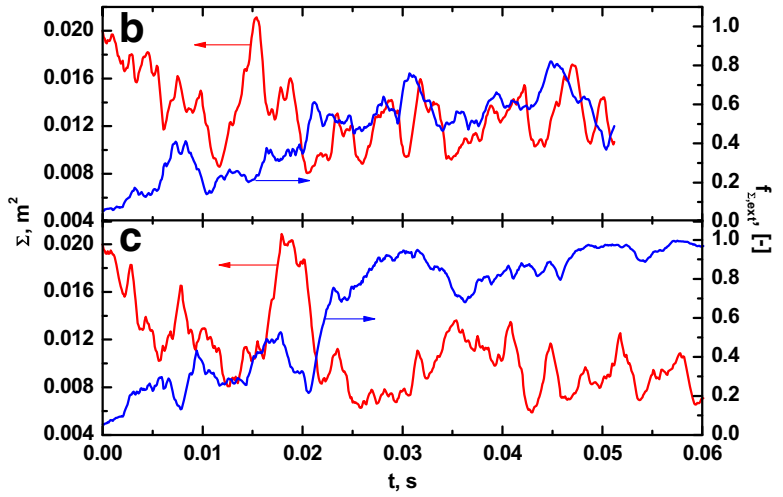

Fig. 7 a Instantaneous contours of stoichiometric mixture fraction from BO2, coloured by conditionally filtered $\mathrm{OH}$ mass fraction at stoichiometry. Blue: low $\mathrm{OH}$ mass fraction and red: high $\mathrm{OH}$ mass fraction. Time records of filtered stoichiometric mixture fraction iso-surface area (red lines) and extinguished fraction (blue lines) from $\mathbf{b ~ S} 3$ and $\mathbf{c} \mathrm{BO} 2$ cases 
a sharp decrease of $f_{\Sigma, \text { ext }}$ occurs occasionally (e.g. $t=0.008$ and $0.02 \mathrm{~s}$ in Fig. $7 \mathrm{c}$ ) probably due to the topological change (e.g. expansion, fragmentation and corrugation) and/or re-ignition of the $\xi_{s t}$ iso-surfaces. The differences in $f_{\Sigma, e x t}$ between a stable flame, where $f_{\Sigma, \text { ext }}$ stays above zero but below unity, and the blow-off case, where $f_{\Sigma, \text { ext }}$ progressively reaches unity, are evident in Fig. 7.

For the blow-off event BO2, Fig. 8 further shows the time records of the extinguished fraction $f_{\Sigma, e x t}$ and $\xi_{s t}$ iso-surface area $\Sigma$ corresponding to different flame sections along the streamwise distance, i.e. flame root $(0<x \leq 0.015 \mathrm{~m})$, middle $(0.015 \mathrm{~m}<x \leq$ $0.03 \mathrm{~m})$ and top $(x>0.03 \mathrm{~m})$, to understand their individual behaviors during the blow-off transient. Here $f_{\Sigma, e x t}$ is calculated with Eq. 19 but based on the extinguished and total $\xi_{s t}$ iso-surface for individual sections. It is seen from Fig. 8a that at $t=0$ the flame root has the largest extinguished fraction while the flame top the weakest. The extinguished fraction $f_{\Sigma, \text { ext }}$ of the three sections increases until about $t=0.03 \mathrm{~s}$ and after that all the three extinguished fractions are close to unity, indicating almost complete extinction occurring on the surface. This is consistent with the results in Fig. 7c. Similar to Fig. 7, the evolutions of $\Sigma$ for each section are also shown in Fig. 8b. Explicitly, $\Sigma$ of the flame bottom has negligible variations during the whole time records. As such, its increase (e.g. at $t=0.03$ s) or decrease (e.g. at $t=0.035 \mathrm{~s}$ ) of $f_{\Sigma, e x t}$ is basically caused by the production or destruction of $\mathrm{OH}$ in mixture fraction space on the iso-surface. However, for the middle section, the strong fluctuations of $\Sigma$ do not lead to the exactly corresponding change of $f_{\Sigma, \text { ext }}$. This is particularly true after $t=0.035 \mathrm{~s}: f_{\Sigma, \text { ext }}$ evolves monotonically towards unity, indicating the gradually severe extinguishment at the middle section. Differently, at the top, $\Sigma$ fluctuates with comparatively low frequency and the simultaneous low $f_{\Sigma, e x t}$ can be seen corresponding to the peak of $\Sigma$, e.g. $t=0.035 \mathrm{~s}$ and $0.041 \mathrm{~s}$. Generally, during the blow-off process of $\mathrm{BO} 2$, local extinction and re-ignition always occur at the bottom and top sections until the onset of complete extinction. This blow-off mode is shared by all

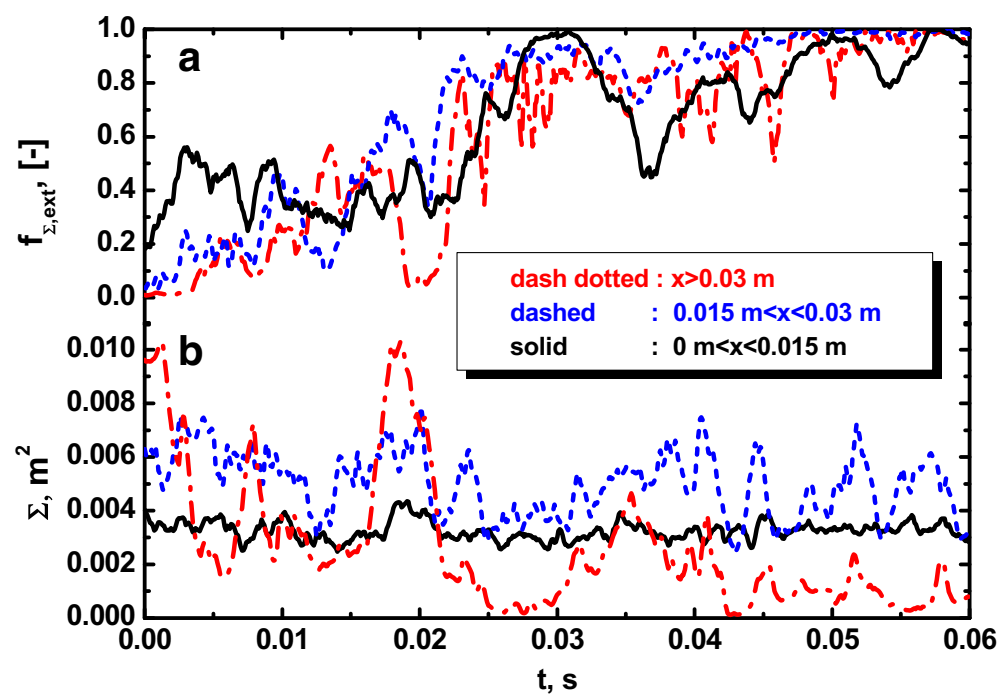

Fig. 8 Time records of $\mathbf{a}$ extinguished fraction and $\mathbf{b} \xi_{s t}$ iso-surface area from BO2 case for root $(0 \mathrm{~m}$ $<x \leq 0.015 \mathrm{~m})$, middle $(0.015 \mathrm{~m}<x \leq 0.03 \mathrm{~m})$ and top $(x>0.03 \mathrm{~m})$ sections of stoichiometric mixture fraction iso-surfaces 

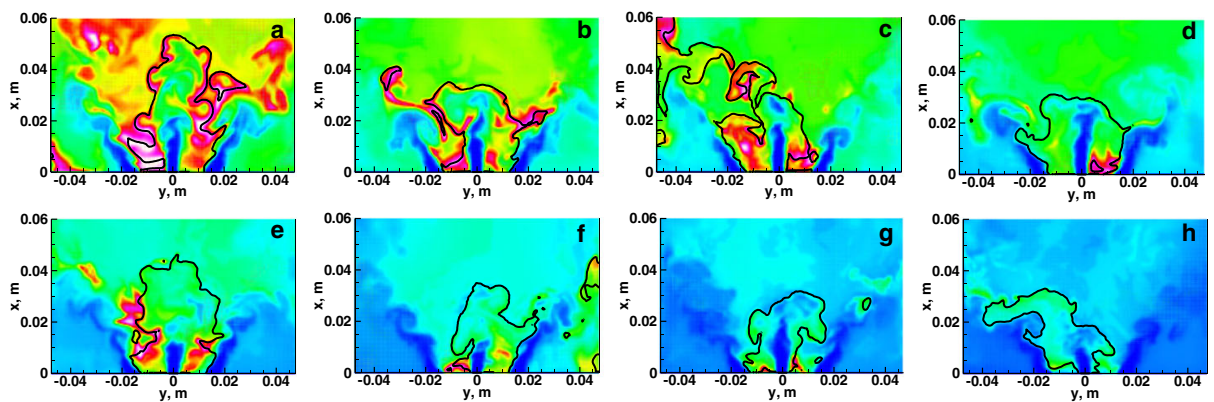

(K) $300 \quad 700 \quad 1100 \quad 1500 \quad 1900$

Fig. 9 Time sequence of distributions of filtered temperature in xoy plane from BO2: $\mathbf{a} t=0 \mathrm{~s}, \mathbf{b} t=0.009 \mathrm{~s}$, $\mathbf{c} t=0.018 \mathrm{~s}, \mathbf{d} t=0.027 \mathrm{~s}, \mathbf{e} t=0.036 \mathrm{~s}, \mathbf{f} t=0.045 \mathrm{~s}, \mathbf{g} t=0.054 \mathrm{~s}$ and $\mathbf{h} t=0.061 \mathrm{~s}$. Lines: instantaneous stoichiometric mixture fraction

the cases marked along $1.25 U_{B O, \exp }$ and $1.6 U_{B O, \exp }$ lines in Fig. 3 due to the same swirl number $S_{N}$.

\subsection{Evolutions of species and scalar dissipation during the blow-off transient}

The contours of filtered temperature $\tilde{T}$ on $x o y$ plane from the BO2 case are plotted in Fig. 9. The eight sub-figures correspond to $t=0-0.061 \mathrm{~s}$, spanning the whole transient blow-off process. At $t=0$ in Fig. 9a, high $\tilde{T}$ appears along the instantaneous $\xi_{s t}$ iso-lines as well as in the downstream of the chamber. Within the subsequent $0.018 \mathrm{~s}$ or so in Fig. $9 \mathrm{~b}$ and c, $\tilde{T}$ becomes weak in the downstream but along the $\xi_{s t}$ iso-lines it is still high, indicating that there are still possible reactions occurring. As the BO2 flame gradually blows off, the filtered temperature $\tilde{T}$ is considerably reduced and only some pockets near the bluff body still exist, as shown in Fig. 9d-g. At $t=0.061 \mathrm{~s}$ in Fig. 9h when the blow-off occurs, the flows in the whole chamber are cooled with $\tilde{T}$ basically less than $700 \mathrm{~K}$.

Corresponding to exactly the same time instants as those in Fig. 9, the contours of filtered $\mathrm{OH}$ mass fraction $\widetilde{Y_{O H}}$ visualized from $\mathrm{BO} 2$ are plotted in Fig. 10. Initially, along the highly
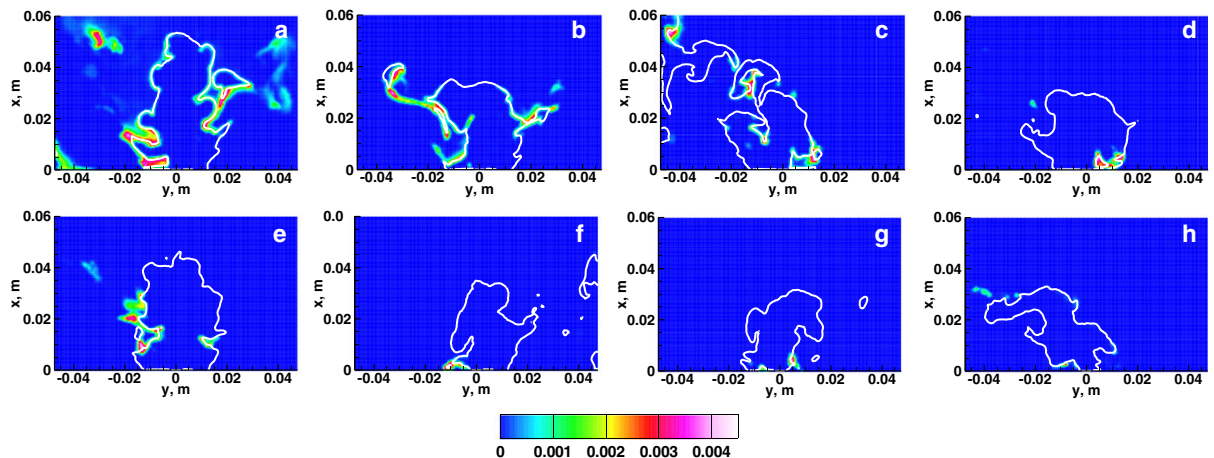

Fig. 10 Time sequence of distributions of filtered $\mathrm{OH}$ mass fraction in xoy plane from $\mathrm{BO} 2$. The temporal information for $\mathbf{a}-\mathbf{h}$ is the same as that in Fig. 9. Lines: instantaneous stoichiometric mixture fraction 
convoluted $\xi_{s t}$ iso-lines, considerable $\widetilde{Y_{O H}}$ can be observed at $t=0$ in Fig. 10a. Meanwhile, regions with low $\widetilde{Y_{O H}}$ corresponding to instantaneous localized extinction exist around the fuel jet tip as well as the right branch near the bluff body edge. Then the extinction extent gradually increases with time from Fig. $10 \mathrm{~b}$ to h. When BO2 blows off at $\tau_{B O} \approx 0.057 \mathrm{~s}$, very little $\mathrm{OH}$ exists in Fig. 10h. Furthermore, re-ignition with local increase of $\mathrm{OH}$ can be seen in the RZ close to the bluff body, quantified by the localized pockets with high $\widetilde{Y_{O H}}$ in Figs. 10d-g. Also, no continuous lift-off near the bluff body surface is seen before complete blow-off, consistent with experiment [15] and similar to other findings in blow-out of unconfined swirl-stabilized non-premixed flames [22]. However, this is different from the phenomenological observations of blow-out process in turbulent non-premixed jet fame, in which case the flame base is continuously pushed far from the burner and the extinction happens between the flame base and burner exit [17]. As such, the blow-off transient in Fig. 10 agrees qualitatively well with the experimental results with OH-PLIF [15, 52].

The time evolution of filtered formaldehyde $\left(\mathrm{CH}_{2} \mathrm{O}\right)$ mass fraction $\widehat{\mathrm{Y}_{\mathrm{CH} 2 \mathrm{O}}}$ from $\mathrm{BO} 2$ is demonstrated in Fig. 11. $\mathrm{CH}_{2} \mathrm{O}$ is a key intermediate from the methyl $\left(\mathrm{CH}_{3}\right)$ oxidation towards $\mathrm{CO}$ and $\mathrm{CO} 2$ and therefore viewed as a significant indicator for the inherent change of flame chemical kinetics, particularly when the finite rate chemistry effects become dominant [56-59]. In the present LES of blow-off in BO2 case, initially when the flame has strong reactivity in Fig. 11a, very few localized $\widehat{Y_{C H 2 O}}$ peaks appear along the $\xi_{s t}$ iso-lines and generally $\mathrm{CH}_{2} \mathrm{O}$ distribution is uniform, say $\widehat{Y_{C H 2 O}} \approx 0.0002$, within the $\xi_{\text {st }}$ envelope. As the flame gradually approaches the global extinction, $\mathrm{CH}_{2} \mathrm{O}$ is considerably accumulated in the IRZ close to the bluff body $(0<x<0.02 \mathrm{~m})$ which can be clearly found from Fig. $11 \mathrm{~b}$ to $\mathrm{f}$. In addition, $\mathrm{CH}_{2} \mathrm{O}$ is intermittently transported from the IRZ downstream towards the chamber walls, e.g. in Fig. 11c and f. The localized high $\mathrm{CH}_{2} \mathrm{O}$ pockets gradually diminish close to the bluff body surface and at $t=0.061 \mathrm{~s}$ when $\mathrm{BO} 2$ is globally extinguished, such pockets basically disappear. However, $\mathrm{CH}_{2} \mathrm{O}$ still has finite concentration in most of the chamber. The $\mathrm{CH}_{2} \mathrm{O}$ evolutions are different from those of $\mathrm{OH}$ shown in Fig. 11, which are also found in in [56, 58, 59].

Figure $12 \mathrm{a}-\mathrm{h}$ shows the evolutions of conditionally filtered $\mathrm{OH}$ mass fraction $\widehat{Y_{O H} \mid \xi_{s t}}$ in the LES resolution during the blow-off transients and their temporal information exactly corresponds to that in Figs. 9-11. Compared to Fig. 10, one can clearly see that the reactive regions in mixture fraction space (i.e. high $\widehat{Y_{O H} \mid \xi_{s t}}$ in Fig. 12) are more distributed than
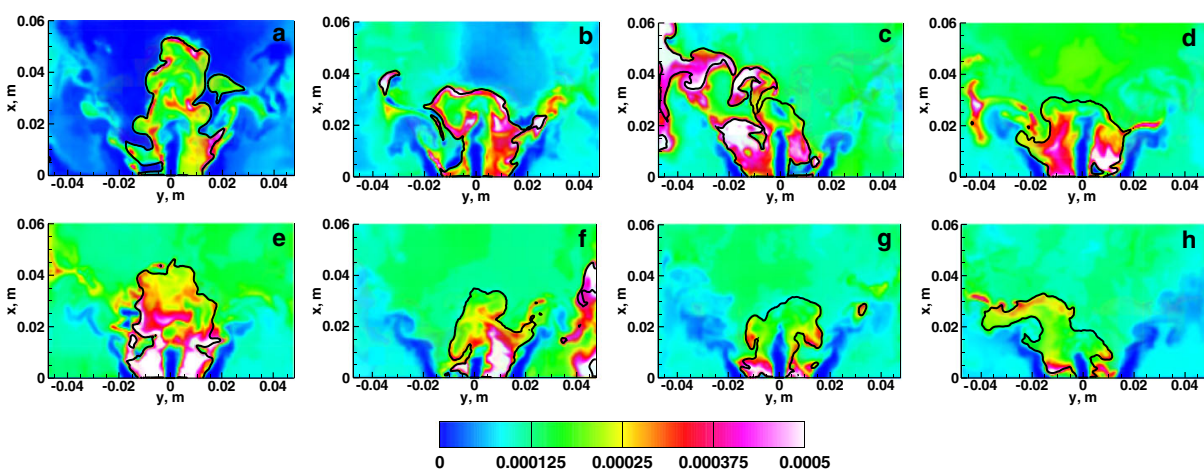

Fig. 11 Time sequence of distributions of filtered $\mathrm{CH}_{2} \mathrm{O}$ mass fraction in xoy plane from $\mathrm{BO} 2$. The temporal information for $\mathbf{a}-\mathbf{h}$ is the same as that in Fig. 9. Lines: instantaneous stoichiometric mixture fraction 

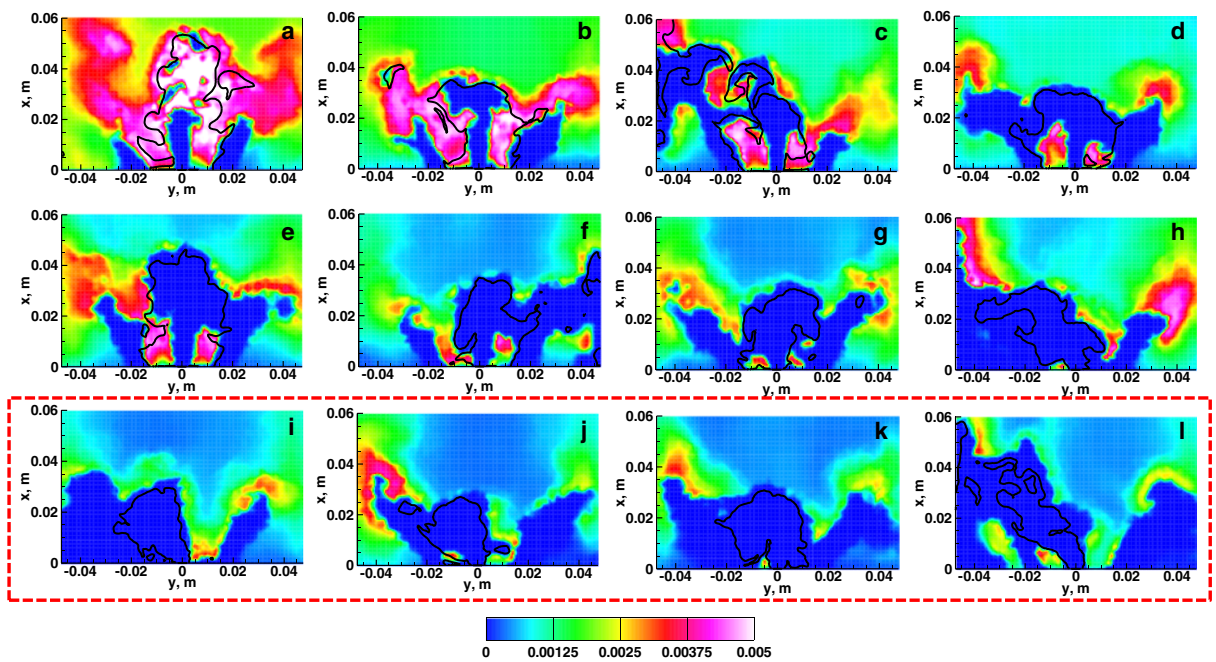

Fig. 12 Time sequence of distributions of conditionally filtered $\mathrm{OH}$ mass fraction at stoichiometry in xoy plane from BO2. Temporal information for $\mathbf{a}-\mathbf{h}$ is same as that for $(\mathbf{a}-\mathbf{h})$ of Fig. 9. The time for $\mathbf{i}-\mathbf{l}$ highlighted by the dashed box is $t=0.063 \mathrm{~s}, 0.072 \mathrm{~s}, 0.081 \mathrm{~s}$ and $0.09 \mathrm{~s}$ respectively, denoting the evolution of conditionally filtered $\mathrm{OH}$ mass fraction in mixture fraction space after the global extinction has been reached in physical space. Lines: instantaneous stoichiometric mixture fraction

those in physical space (i.e. high $\widetilde{Y_{O H}}$ in Fig. 10). Regions with low $\widetilde{Y_{O H} \mid \xi_{s t}}$ only exist in the central fuel jet and swirling air inlet when $t=0$ and $0.0009 \mathrm{~s}$ in Fig. 12a and b, respectively. This directly results from the convection of inert CMC solutions from both inlets as the boundary conditions. In contrast, high $\widehat{Y_{O H} \mid \xi_{s t}}$, indicating the full reactivity in $\eta$-space, can be seen in the IRZ close to the bluff body. In Fig. 12c and d, it can be seen that high $\widehat{Y_{O H} \mid \xi_{s t}}$ regions are disconnected from the downstream reactive regions and become isolated in this two-dimensional slice. Nevertheless, in Fig. 12e and $\mathrm{f}$ the left high $\widehat{Y_{O H} \mid \xi_{s t}}$ region expands and then coalesces with the downstream zones, leading to the onset of re-ignition there as demonstrated in Fig. 12e and f. The extinction regions continuously increase in size in the last stage of blow-off in Fig. 12g and h. When the flame is extinguished, $\widehat{Y_{O H} \mid \xi_{s t}}$ is zero almost along the whole $\xi_{s t}$ iso-lines.

Figure 12i-1 correspond to the evolutions of $\widehat{Y_{O H} \mid \xi_{s t}}$ during the post-extinction stage (approximately $0.03 \mathrm{~s}$ more after blow-off). There are always some regions with finite residual $\widehat{Y_{O H} \mid \xi_{s t}}$, e.g. around the downstream of the air stream and immediately outside the $\xi_{s t}$ iso-lines. Complete quenching of $\mathrm{OH}$ in mixture fraction space $\widetilde{Y_{O H} \mid \xi_{s t}}$ does not occur, although from Fig. $10 \widetilde{Y_{O H}}$ is zero in the entire chamber after blow-off. This inconsistency can be analyzed as follows: (i) residual $\widehat{Y_{O H} \mid \xi_{s t}}$ regions may be never quenched (e.g. around $x=y=0.04 \mathrm{~m}$ ) and/or obtain $\mathrm{OH}$ through the neighboring CMC cells in the different directions, particularly due to the existence of the strong recirculating flows in IRZ (e.g. $0<y<0.01 \mathrm{~m}$ close to the bluff body shown in Fig. 12i and 1) and (ii) the scalar dissipation in these regions is relatively low so that burning conditional reactive scalars are difficult to reach extinction state. After blow-off, another interesting phenomenon indicated by Fig. $12 \mathrm{i}-1$ is that the $\xi_{s t}$ iso-lines and the enclosed basically have zero $\widehat{Y_{O H} \mid \xi_{s t}}$, even 
if the immediately neighboring cells have high values. This ensures the global extinction along the $\xi_{s t}$ iso-lines in physical space, as shown in Fig. 10. However, very localized and weak $\widehat{Y_{O H} \mid \xi_{s t}}$ would still appear along $\xi_{s t}$ iso-lines after blow-off (e.g. $y=-0.01 \mathrm{~m}$ close to the bluff body in Fig. 12j) but this would not ignite the whole or a large fraction of the $\xi_{s t}$ iso-lines any more. Generally, the above mentioned phenomenon is expected to be related to the evolution of scalar dissipation rate and will be discussed later in this Section 3.4.

Essentially, three-dimensional flow effects on flame re-ignition exist in turbulent jet flames $[7,60]$, let alone in swirling flows with strong tangential motion. Indeed, they have been observed in the measurements of the current investigated swirl flames [15] and also appears in a partially pre-mixed swirl burner [61]. In the context of the CMC model, the three-dimensional flow effects occurring between neighboring CMC cells can be predicted by the conditional convection, dilatation and sub-grid diffusion terms in Eq. 5. Based on the evolutions of $\widehat{Y_{O H} \mid \xi_{s t}}$ on the two-dimensional slice shown in Fig. 12, there are mainly two modes of CMC cell interactions. First, the coalescence of reaction zones with high $\widetilde{Y_{O H} \mid \xi_{s t}}$ (as shown in Fig. 12) and appearance of isolated $\widetilde{Y_{O H} \mid \xi_{s t}}$ pockets (not shown in Fig. 12) in the IRZ are caused by transporting the fully burning conditional profiles between neighboring cells (particularly from downstream cells due to flow reverse). Second, at the downstream of the swirling air streams and close to the chamber walls (spanwise distance $y= \pm 0.04 \mathrm{~m}$ and streamwise distance $x=0.04 \mathrm{~m}$ ), burning profiles in $\eta$-space, e.g. high $\widehat{Y_{O H} \mid \xi_{s t}}$ in Fig. 12, are intermittently from the IRZ due to the strong shearing between the swirling air stream and IRZ. The significance of correctly predicting the re-ignition at the two above modes by the CMC model would be clear when the time series of $Q$ for BO2 in Fig. 4b is re-visited. For instance, the intermittent peaks of total heat release rate $Q$ for BO2 at approximately $t=0.02$ are attributed to the second mode while the long
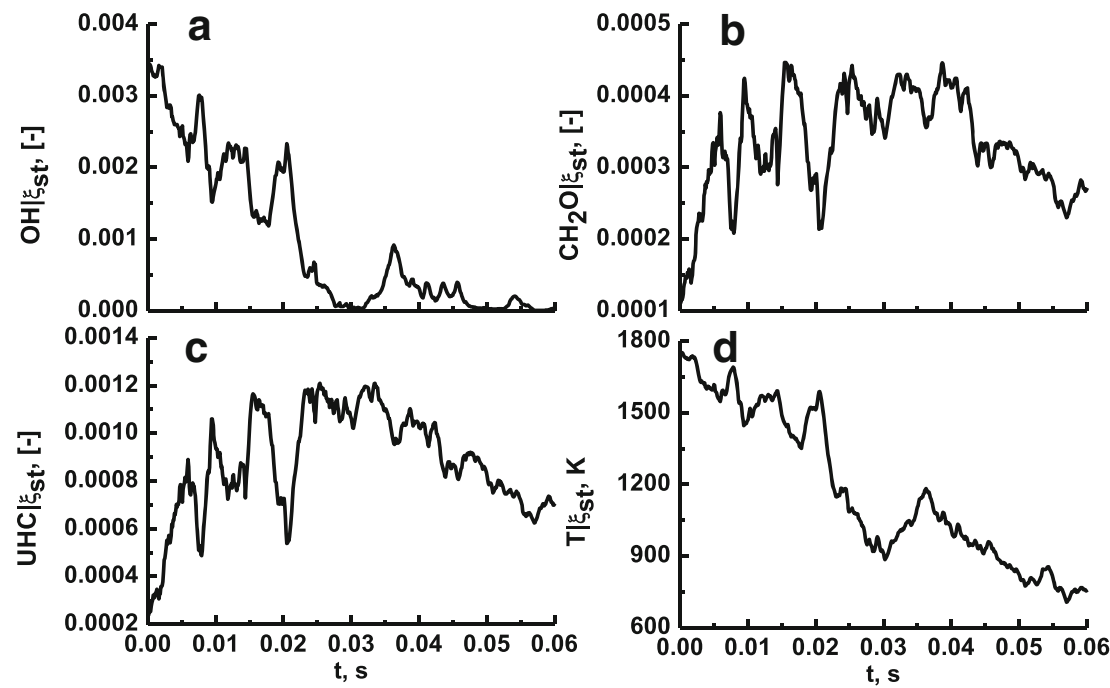

Fig. 13 Time evolutions of the surface-averaged conditional stoichiometric mass fractions of $\mathbf{a} \mathrm{OH}, \mathbf{b} \mathrm{CH}_{2} \mathrm{O}$, c unburned $\mathrm{C}_{2}$ hydrocarbons and temperature from BO2. The averaging is performed for each time instant based on the samples on the filtered $\xi_{s t}$ iso-surfaces 
persistence with low $Q$ the first one. The ability to predict transport effects in physical space by the CMC model was also pointed out when the upstream extinction occurring in the Calgary lifted flames [62], localized extinction in Sandia flame F [34], TECFLAM [63] and the swirl non-premixed flames [35] are investigated.

Figure 13 presents the time evolution of conditional mass fractions $\left\langle\widetilde{Y_{a} \mid \xi_{\text {st }}}\right\rangle$ and temperature $\left\langle\widetilde{T \mid \xi_{\mathrm{st}}}\right\rangle$ at stoichiometry from the blow-off event BO2. Here the results are spatially averaged based on the samples extracted from $\xi_{s t}$ iso-surfaces for each time instant. During the whole simulated period, $\left\langle\widehat{Y_{O H} \mid \xi_{\text {st }}}\right\rangle$ gradually decreases toward blow-off at $t=0.057$ $\mathrm{s}$, as shown in Fig. 13a. From Fig. $13 \mathrm{~b}$ and c, the surface averaged $\mathrm{CH}_{2} \mathrm{O}$ and unburned $\mathrm{C}_{2}$ hydrocarbons (UHC, i.e. ethane $\mathrm{C}_{2} \mathrm{H}_{6}$, ethylene $\mathrm{C}_{2} \mathrm{H}_{4}$ and acetylene $\mathrm{C}_{2} \mathrm{H}_{2}$ in ARM2 mechanism) concentrations first increases and after $t=0.02 \mathrm{~s}$ decrease. At $t=0.06 \mathrm{~s}$ which is very close to complete blow-off, they are still around $50 \%$ of the individual peaks during the time series. The averaged temperature $\left\langle\widetilde{T \mid \xi_{\text {st }}}\right\rangle$ in Fig. 13d presents a similar change to that of $\left\langle\widehat{Y_{O H} \mid \xi_{\mathrm{st}}}\right\rangle$ in Fig. 13a.

The distributions of filtered scalar dissipation rate $\tilde{N}$ in logarithmic scale corresponding to the blow-off case BO2 are presented in Fig. 14. At $t=0$ shown in Fig. 14a, the majority of high filtered scalar dissipation is spatially confined to thin layers located immediately around the central fuel jet upstream (i.e. $0<x \leq 0.015 \mathrm{~m}$ ) while downstream (i.e. 0.015 $\mathrm{m}<x<0.055 \mathrm{~m}$ ) the layer structures mostly follow the instantaneous $\xi_{s t}$ iso-lines. These
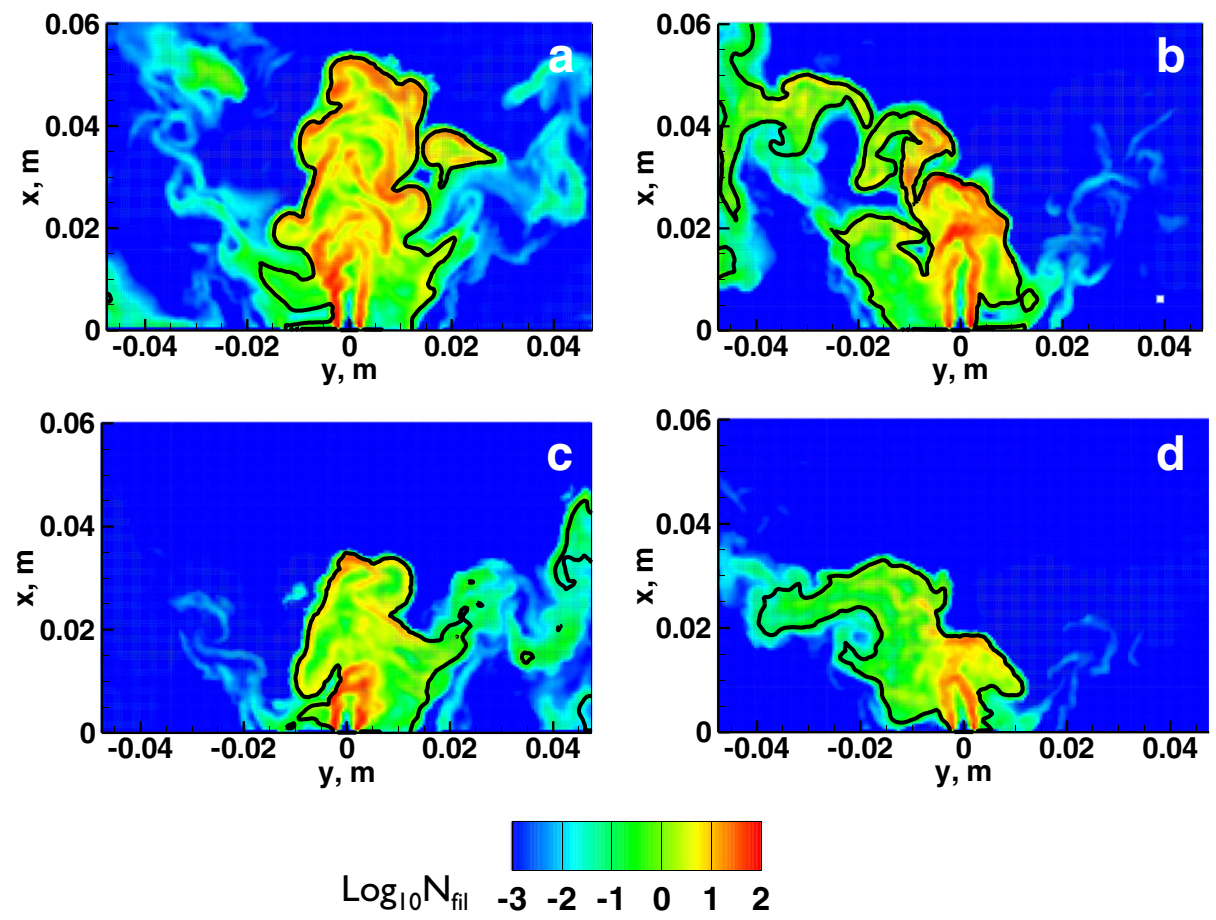

Fig. 14 Distributions of filtered scalar dissipation at $\mathbf{a} t=0 \mathrm{~s}, \mathbf{b} t=0.018 \mathrm{~s}, \mathbf{c} t=0.045 \mathrm{~s}$, and $\mathbf{d} t=0.061$ $\mathrm{s}$ from $\mathrm{BO} 2$. Lines: instantaneous stoichiometric mixture fraction 
scalar dissipation distributions qualitatively resemble those in turbulent jet flames $[9,64$, 65]. At $t=0.018 \mathrm{~s}$ and $0.045 \mathrm{~s}$ in Fig. $14 \mathrm{~b}$ and $\mathrm{c}$, two main features can be observed. First, the layer-like structures there gradually become weak. Second, small pockets with the intermediate $\tilde{N}$ are alternately transported toward the chamber side walls accompanied by the severe disintegration of the $\xi_{s t}$ iso-surfaces there. This phenomenon is manifested by the intermittent occurrences of local extinction and re-ignition near the chamber walls, as shown in Figs. 12e, g and h. At the blow-off point $t=0.061 \mathrm{~s}$ in Fig. 14d, the fuel jet penetrates less. If Fig. $12 \mathrm{~h}$ is re-visited with Fig. 14d, one can see that, after blow-off, extinction in mixture fraction space occurs almost in the entire regions with finite $\tilde{N}$, which are roughly enclosed by the $\xi_{s t}$ iso-lines. However, beyond the $\xi_{s t}$ iso-lines where $\tilde{N}$ is small, there are still considerable $\widehat{Y_{O H} \mid \xi_{s t}}$. Such relation between $\tilde{N}$ and $\widetilde{Y_{O H} \mid \xi_{s t}}$ under blowoff condition is not observed when the flame is strongly reactive if the similar comparison between Figs. 14a and 12a is made. The evolutions of the filtered scalar dissipation structures are expected to be affected by the changes of the turbulence when the flame gradually approaches blow-off.

The averaged filtered and conditionally filtered stoichiometric scalar dissipation, i.e. $\tilde{N}$ and $\widetilde{N \mid \xi_{s t}}$, on the $\xi_{s t}$ iso-surfaces are calculated and their time series are plotted in Fig. 15. Here the averaging procedure is same as that used in Fig. 13. Figure 15a presents the time series of surface-averaged $\tilde{N}$ and $\widehat{N \mid \xi_{s t}}$ from the blow-off case BO2. Generally, the averaged $\tilde{N}$ decreases with time. $\widehat{N \mid \xi_{s t}}$ is well below the conditional scalar dissipation at extinction from a stand-alone $0 \mathrm{D}-\mathrm{CMC}$ calculation, which is $18.31 / \mathrm{s}$, and shows little change before $t=0.05 \mathrm{~s}$. However, at the last stage of blow-off (e.g. $t=0.05-0.06 \mathrm{~s}$ in Fig. 15a), the peaky fluctuations with high frequency and large magnitudes appear, which is a prominent symptom of the flame close to blow-off. For comparison, the corresponding results of another blow-off case with larger air bulk velocity (BO3) and a stable flame

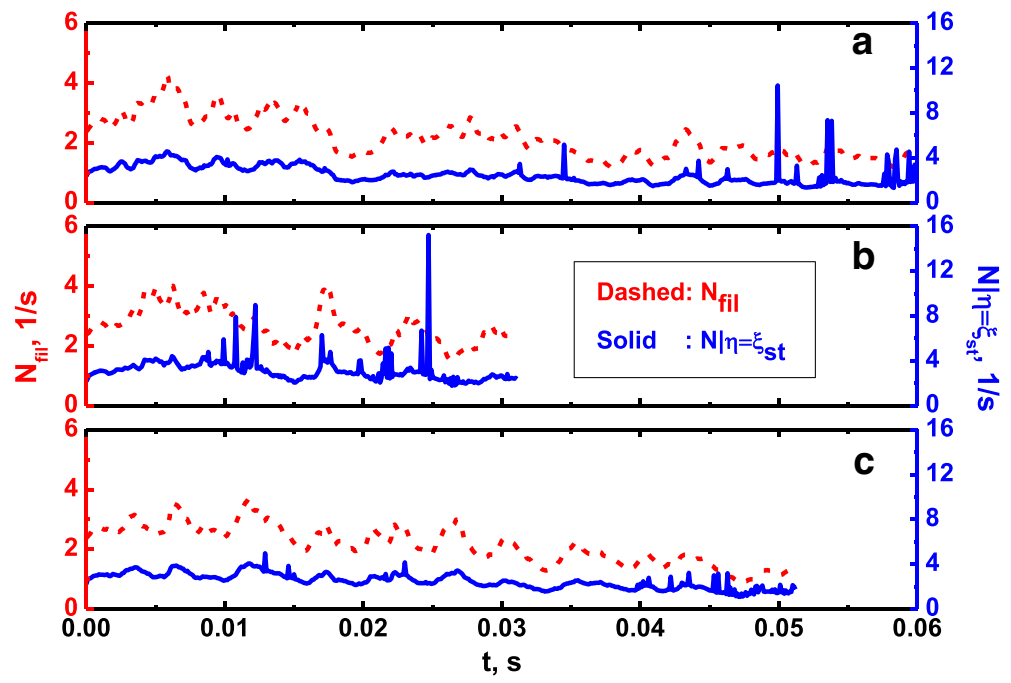

Fig. 15 Time series of the surface-averaged unconditionally filtered (dashed lines) and conditionally filtered stoichiometric (solid lines) scalar dissipation from cases of a BO2, b BO3 and $\mathbf{c} \mathrm{S} 3$. The averaging is performed for each time instant based on the samples on the filtered $\xi_{s t}$ iso-surfaces 
case (S3) are also presented in Fig. 15b and c. One can clearly see that for BO3 the highfrequency fluctuations of $\widehat{N \mid \xi_{s t}}$ starts at $t=0.01 \mathrm{~s}$, much earlier than BO1 case in Fig. 15a. However, for S3, the fluctuation magnitudes are comparatively small.

\subsection{Blow-off time}

The blow-off time $\tau_{B O}$ of $\mathrm{BO} 1, \mathrm{BO} 2$ and $\mathrm{BO} 3$ has been demonstrated in Fig. 4 based on the time records of total heat release rate $Q$ and it is shown that $\tau_{B O}$ for BO1 which is on $1.25 U_{B O, \text { exp }}$ curve is higher than that for $\mathrm{BO} 3$ on $1.6 U_{B O \text {,exp }}$ curve by around $45 \%$. Figure 16 plots the time series of total heat release rate of all the cases on both $1.25 U_{B O \text {,exp }}$ and $1.6 U_{B O, \exp }$ curves in Fig. 4. Based on Fig. 16a-e, basically $\tau_{B O}$ of $1.6 U_{B O, \exp }$ curves is smaller than that of $1.25 U_{B O, \exp }$ curve. However, the exceptional circumstance can be seen when $U_{f}=34.3 \mathrm{~m} / \mathrm{s}$ and the blow-off time $\tau_{B O}$ for both cases is approximately the same, i.e. $\tau_{B O}=0.04 \mathrm{~s}$. In addition, the blow-off time of the cases on $1.25 U_{B O \text {,exp }}$ and $1.6 U_{B O, \exp }$ curve demonstrates the non-monotonic behaviors with simultaneously increased fuel and air bulk velocities. For example, on $1.25 U_{B O \text {,exp }}$ curve, $\tau_{B O}$ for cases with $U_{f}=18.7 \mathrm{~m} / \mathrm{s}, 29.2 \mathrm{~m} / \mathrm{s}$ and $39.5 \mathrm{~m} / \mathrm{s}$ is about $0.07 \mathrm{~s}, 0.06 \mathrm{~s}$ and $0.05 \mathrm{~s}$, but for $U_{f}=24 \mathrm{~m} / \mathrm{s}$ and $34.3 \mathrm{~m} / \mathrm{s}$, it is around $0.04 \mathrm{~s}$. Actually the longer blow-off time is caused by the existence of small flame pockets in the IRZ when the flame is very close to blow-off, which is quantified by the long-lasting low $Q$, e.g. shown in Fig. 16a. This phenomenon is

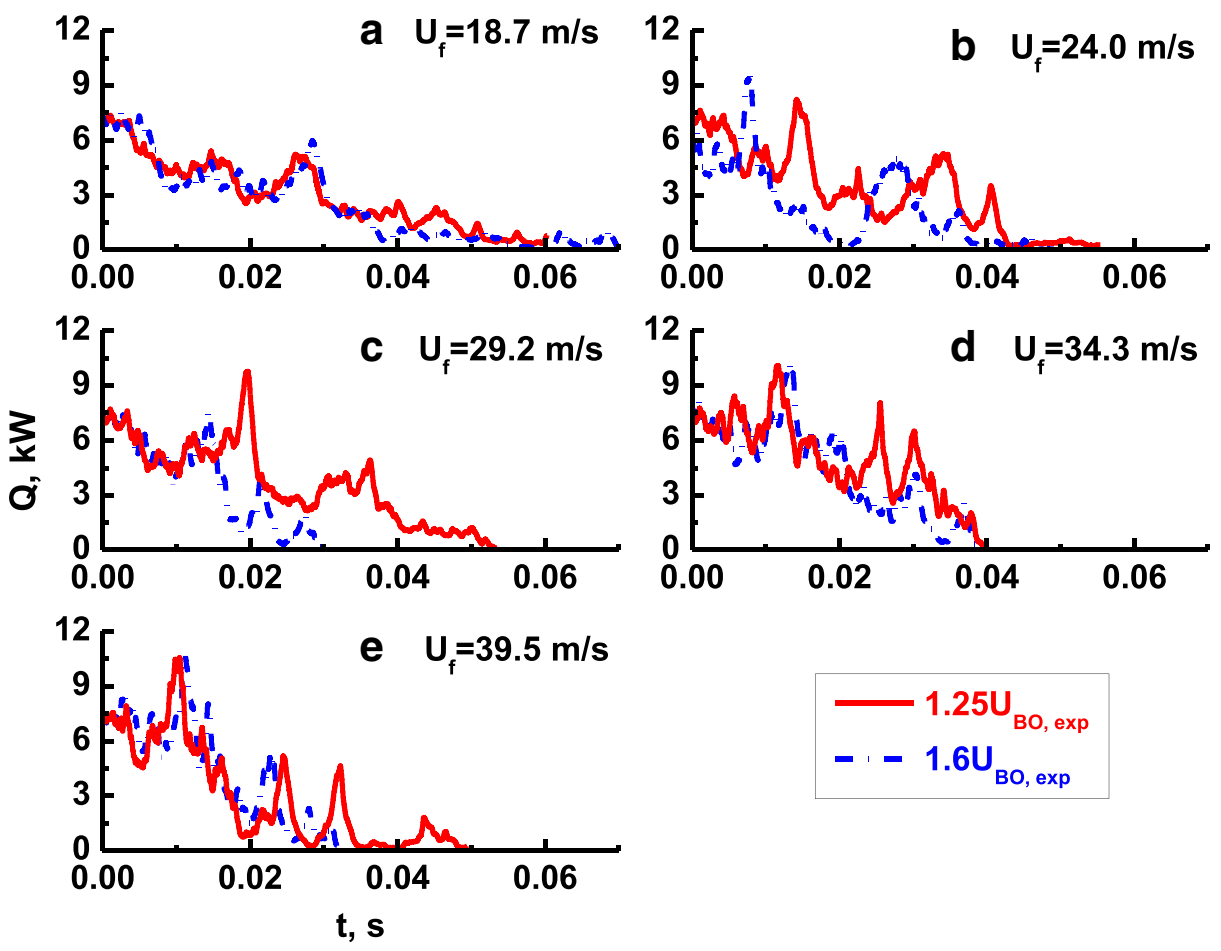

Fig. 16 Time records of total heat release from the blow-off cases along the curves of $1.25 U_{B O, \exp }$ and $1.6 U_{B O, \exp }$ shown in Fig. 3 
not observed in the cases on $1.6 U_{B O \text {,exp }}$ curve except the one with $U_{f}=18.7 \mathrm{~m} / \mathrm{s}$ presented in Fig. 16a.

It should be highlighted that the evolutions of the total heat release rates in Fig. 16 are from a single LES/3D-CMC simulation for the corresponding operating conditions and therefore the blow-off time indicated by Fig. 16 does not have statistical sense. To generalize the variations of blow-off time with respect to air and/or fuel bulk velocities, the efforts to predict their mean should be made, which is not conducted in the present investigations due to the prohibitive computational cost. Cavaliere et al. did not measure $\tau_{B O}$ for other cases either, except for S2 which has been discussed above [15]. Understanding the correlation between the blow-off time and a range of operating conditions possesses the practical significance for designing the new burner and combustor and therefore further computational or experimental studies are needed.

\section{Conclusions}

The LES/3D-CMC model with detailed chemistry is employed to predict the blow-off conditions and dynamics of swirl-stabilized non-premixed methane flames. The blow-off range from the simulations was obtained and the critical air bulk velocity from the present simulations was larger than the experimental value by around $25 \%$, which shows the good predictive capability of the LES/3D-CMC. This confirms the capability of the sub-grid CMC model in predicting the strong turbulence-chemistry interaction and this level of agreement is promising and might at present even be considered acceptable from an engineering perspective. The results show that during the blow-off process the total heat release gradually decreases with pronounced fluctuations. The predicted blow-off transient lasts a time in good agreement with the experimental results. The extinguished fraction was introduced to measure the degree of extinction occurrence on the iso-surfaces of the filtered stoichiometric mixture fraction. The extinguished fraction gradually increased and reached unity when the flame approached the blow-off point. Close to blow-off, high-frequency and high-magnitude fluctuations of the conditionally filtered stoichiometric scalar dissipation rate on the iso-surfaces of the filtered stoichiometric mixture fraction were evident.

Acknowledgments This work was financially supported by Engineering and Physical Sciences Research Council (EPSRC) and Rolls-Royce through a Dorothy Hodgkin Postgraduate Award. This work used the computational resources from ARCHER cluster of the UK National Supercomputing Service (http://www. archer.ac.uk) under the project of United Kingdom Consortium on Turbulent Reacting Flows (UKCTRF) and the Darwin Supercomputer of the University of Cambridge High Performance Computing Service (http:// www.hpc.cam.ac.uk/).

Open Access This article is distributed under the terms of the Creative Commons Attribution 4.0 International License (http://creativecommons.org/licenses/by/4.0/), which permits unrestricted use, distribution, and reproduction in any medium, provided you give appropriate credit to the original author(s) and the source, provide a link to the Creative Commons license, and indicate if changes were made.

\section{References}

1. Poinsot, T., Veynante, D.: Theoretical and Numerical Combustion. R. T. Edwards, Philadephia (2005)

2. Law, C.K.: Combustion Physics. Cambridge University Press, New York (2006) 
3. Meier, W., Barlow, R.S., Chen, Y.-L., Chen, J.-Y.: Raman/Rayleigh/LIF measurements in a turbulent $\mathrm{CH}_{4} / \mathrm{H}_{2} / \mathrm{N}_{2}$ jet diffusion flame: experimental techniques and turbulence-chemistry interaction. Combust. Flame 123, 326-343 (2000)

4. Barlow, R.S., Frank, J.H.: Effects of turbulent on species mass fractions in methane/air jet flames. 27th Symposium (International) on Combustion 27, 1087-1095 (1998)

5. Masri, A.R., Dibble, R.W., Bilger, R.W.: Turbulent nonpremixed flames of methane near extinction: mean structure from Raman measurements. Combust. Flame 71, 245-266 (1988)

6. Hult, J., Meier, U., Meier, W., Harvey, A., Kaminski, C.F.: Experimental analysis of local flame extinction in a turbulent jet diffusion flame by high repetition 2-D laser techniques and multi-scalar measurements. Proc. Combust. Inst. 30, 701-709 (2005)

7. Steinberg, A.M., Boxx, I., Arndt, C.M., Frank, J.H., Meier, W.: Experimental study of flame-hole reignition mechanisms in a turbulent non-premixed jet flame using sustained multi-kHz PIV and crossed-plane OH PLIF. Proc. Combust. Inst. 33, 1663-1672 (2011)

8. Kaiser, S.A., Frank, J.H.: Spatial scales of extinction and dissipation in the near field of non-premixed turbulent jet flames. Proc. Combust. Inst. 32, 1639-1646 (2009)

9. Sutton, J.A., Driscoll, J.F.: Measurements and statistics of mixture fraction and scalar dissipation rates in turbulent non-premixed jet flames. Combust. Flame 160, 1767-1778 (2013)

10. Sutton, J.A., Driscoll, J.F.: Imaging of local flame extinction due to the interaction of scalar dissipation layers and the stoichiometric contour in turbulent non-premixed flames. Proc. Combust. Inst. 31, 14871495 (2007)

11. Masri, A.R., Kalt, P.A.M., Barlow, R.S.: The compositional structure of swirl-stabilised turbulent nonpremixed flames. Combust. Flame 137, 1-37 (2004)

12. Meier, W., Duan, X.R., Weigand, P.: Investigations of swirl flames in a gas turbine model combustor: II. Turbulence-chemistry interactions. Combust. Flame 144(1-2), 225-236 (2006)

13. Gicquel, L.Y.M., Staffelbach, G., Poinsot, T.: Large eddy simulations of gaseous flames in gas turbine combustion chambers. Prog. Energy Combust. Sci. 38(6), 782-817 (2012)

14. Stöhr, M., Boxx, I., Carter, C., Meier, W.: Dynamics of lean blowout of a swirl-stabilized flame in a gas turbine model combustor. Proc. Combust. Inst. 33(2), 2953-2960 (2011)

15. Cavaliere, D., Kariuki, J., Mastorakos, E.: A comparison of the blow-off behaviour of swirl-stabilized premixed, non-premixed and spray flames. Flow Turbul. Combust. 91, 347-372 (2013)

16. Masri, A.R., Kalt, P.A.M., Al-Abdeli, Y.M., Barlow, R.S.: Turbulence-chemistry interactions in nonpremixed swirling flames. Combust. Theor. Model. 11, 653-673 (2007)

17. Wu, C.-Y., Chao, Y.-C., Cheng, T.-S., Li, Y.-H., Lee, K.-Y., Yuan, T.: The blowout mechanism of turbulent jet diffusion flames. Combust. Flame 145, 481-494 (2006)

18. Pitts, W.M.: Importance of isothermal mixing processes to the understanding of lift-off and blowout of turbulent jet diffusion flames. Combust. Flame 76, 197-212 (1989)

19. Bradley, D., Gaskell, P.H., Gu, X.J.: The mathematical modeling of liftoff and blowoff of turbulent non-premixed methane jet flames at high strain rates. Symp. Combust. 27, 1199-1206 (1998)

20. Kalghatgi, G.T.: Blow-out stability of gaseous jet diffusion flames. Part I: In still air. Combust. Sci. Tech. 26, 233-239 (1981)

21. Feikema, D., Chen, R.-H., Driscoll, J.F.: Enhancement of flame blowout limits by the use of swirl. Combust. Flame 80, 183-195 (1990)

22. Feikema, D., Chen, R.-H., Driscoll, J.F.: Blowout of nonpremixed flames: Maximum coaxial air velocities achievable, with and without swirl Combust. Flame 86, 347-358 (1991)

23. Al-Abdeli, Y.M., Masri, A.R.: Stability characteristics and flowfields of turbulent non-premixed swirling flames. Combust. Theor. Model. 7, 731-766 (2003)

24. Pierce, C.D., Moin, P.: Progress-variable approach for large-eddy simulation of non-premixed turbulent combustion. J. Fluid Mech. 504, 73-97 (2004)

25. Ihme, M., Pitsch, H.: Prediction of extinction and reignition in nonpremixed turbulent flames using a flamelet/progress variable model 2. Application in LES of Sandia flames D and E. Combust. Flame 155, 90-107 (2008)

26. $\mathrm{Xu}$, J., Pope, S.B.: PDF calculations of turbulent nonpremixed flames with local extinction. Combust. Flame 123, 281-307 (2000)

27. Prasad, V.N., Juddoo, M., Masri, A.R., Jones, W.P., Luo, K.H.: Investigation of extinction and re-ignition in piloted turbulent non-premixed methane-air flames using LES and high-speed OH-PLIF. Combust. Theor. Model. 17, 483-503 (2013)

28. Vogiatzaki, K., Kronenburg, A., Navarro-Martinez, S., Jones, W.: Stochastic multiple mapping conditioning for a piloted, turbulent jet diffusion flame. Proc. Combust. Inst. 33(1), 1523-1531 (2011)

29. Ge, Y., Cleary, M.J., Klimenko, A.Y.: A comparative study of Sandia flame series (D-F) using sparseLagrangian MMC modelling. Proc. Combust. Inst. 34(1), 1325-1332 (2013) 
30. Wandel, A.P., Lindstedt, R.P.: Hybrid multiple mapping conditioning modeling of local extinction. Proc. Combust. Inst. 34(1), 1365-1372 (2013)

31. Cha, C.M., Kosaly, G., Pitsch, H.: Modeling extinction and reignition in turbulent nonpremixed combustion using a doubly-conditional moment closure approach. Phys. Fluids 13, 3824-3834 (2001)

32. Cha, C.M., Pitsch, H.: Higher-order conditional moment closure modelling of local extinction and reignition in turbulent combustion. Combust. Theor. Model. 6, 425-437 (2002)

33. Kronenburg, A., Kostka, M.: Modeling extinction and reignition in turbulent flames. Combust. Flame 143, 342-356 (2005)

34. Garmory, A., Mastorakos, E.: Capturing localised extinction in Sandia Flame F with LES-CMC. Proc. Combust. Inst. 33, 1673-1680 (2011)

35. Zhang, H., Garmory, A., Cavaliere, D.E., Mastorakos, E.: Large Eddy simulation/conditional moment closure modeling of swirl-stabilized non-premixed flames with local extinction. Proc. Combust. Inst. $\mathbf{3 5}$, 1167-1174 (2015)

36. Zhang, H., Mastorakos, E.: Effects of wall heat loss on swirl-stabilized non-premixed flames with localized extinction. In: 10th International ERCOFTAC Symposium on Engineering Turbulence Modelling and Measurements, Marbella, Spain (2014)

37. Dawson, J.R., Gordon, R.L., Kariuki, J., Mastorakos, E., Masri, A.R., Juddoo, M.: Visualization of blowoff events in bluff-body stabilized turbulent premixed flames. Proc. Combust. Inst. 33, 1559-1566 (2011)

38. Kariuki, J., Dawson, J.R., Mastorakos, E.: Measurements in turbulent premixed bluff body flames close to blow-off. Combust. Flame 159, 2589-2607 (2012)

39. Chaudhuri, S., Kostka, S., Renfro, M.W., Cetegen, B.M.: Blowoff dynamics of bluff body stabilized turbulent premixed flames. Combust. Flame 157, 790-802 (2010)

40. Tyliszczak, A., Cavaliere, D.E., Mastorakos, E.: LES/CMC of blow-off in a liquid fueled swirl burner. Flow Turbul. Combust. 92, 237-267 (2014)

41. Fureby, C.: On subgrid scale modeling in large eddy simulations of compressible fluid flow. Phys. Fluids 8, 1301-1429 (1996)

42. Pera, C., Réveillon, J., Vervisch, L., Domingo, P.: Modeling subgrid scale mixture fraction variance in LES of evaporatimg spray. Combust. Flame 146, 635-648 (2006)

43. Jiménez, C., Ducros, F., Cuenot, B., Bédat, B.: Subgrid scale variance and dissipation of a scalar field in large eddy simulations. Phys. Fluids (1994-present) 13(6), 1748-1754 (2001)

44. Pierce, C.D., Moin, P.: A dynamic model for subgrid-scale variance and dissipation rate of a conserved scalar. Phys. Fluids 10, 3041-3044 (1998)

45. Garmory, A., Mastorakos, E.: Numerical simulation of oxy-fuel jet flames using unstructured LESCMC. Proc. Combust. Inst. 35, 1207-1214 (2015)

46. Brien, O.E., Jiang, T.L.: The conditional dissipation rate of an initially binary scalar in homogeneous turbulence. Phys. Fluids 3, 3121-3123 (1991)

47. Triantafyllidis, A., Mastorakos, E.: Implementation issues of the conditional moment closure model in large eddy simulations. Flow Turbul. Combust. 84, 481-512 (2010)

48. Hergart, C., Peters, N.: Applying the representative interactive flamelet model to evaluate the potential effect of wall heat transfer on soot emissions in a small-bore DI Diesel engine. ASME J. Eng. Gas Turbines Power 124, 1042-1052 (2001)

49. De Paola, G., Mastorakos, E., Wright, Y.M., Boulouchos, K.: Diesel engine simulations with multidimensional conditional moment closure. Combust. Sci. Tech. 180, 883-899 (2008)

50. Beer, J.M., Chigier, N.A.: Combustion Aerodynamics. Applied Science Publishers Ltd., London (1971)

51. Zhang, H.: Extinction in Turbulent Swirling Non-premixed Flames. Ph.D. Thesis, University of Cambridge (2015)

52. Cavaliere, D.E.: Blow-off in Gas Turbine Combustors. Ph.D. Thesis, University of Cambridge (2013)

53. Brown, P.N., Hindmarsh, A.C.: Reduced storage matrix methods in stiff ODE systems. J. Comput. Appl. Math. 31, 40-91 (1989)

54. Sung, C.J., Law, C.K., Chen, J.Y.: An augmented reduced mechanism for methane oxidation with comprehensive global parametric validation. Proc. Combust. Inst. 27, 295-304 (1998)

55. Lignell, D., Chen, J., Schmutz, H.: Effects of Damköhler number on flame extinction and reignition in turbulent non-premixed flames using DNS. Combust. Flame 158, 949-963 (2011)

56. Yuan, R., Kariuki, J., Dowlut, A., Balachandran, R., Mastorakos, E.: Reaction zone visualisation in swirling spray n-heptane flames. Proc. Combust. Inst. 35, 1649-1656 (2015)

57. Kariuki, J., Dowlut, A., Yuan, R., Balachandran, R., Mastorakos, E.: Heat release imaging in turbulent premixed methane-air flames close to blow-off. Proc. Combust. Inst. 35, 1443-1450 (2015) 
58. Böckle, S., Kazenwadel, J., kunzelmann, T., Shin, D.-I., Schulz, C., Wolfrum, J.: Simultaneous singleshot laser-based imaging of formaldehyde, $\mathrm{OH}$, and temperature in turbulent flames. Proc. Combust. Inst. 28, 279-286 (2000)

59. Medwell, P., Kalt, P., Dally, B.: Simultaneous imaging of $\mathrm{OH}$, formaldehyde, and temperature of turbulent nonpremixed jet flames in a heated and diluted coflow. Combust. Flame 148, 48-61 (2007)

60. Juddoo, M., Masri, A.R.: High-speed OH-PLIF imaging of extinction and re-ignition in non-premixed flames with various levels of oxygenation. Combust. Flame 158, 902-914 (2011)

61. Boxx, I., Stöhr, M., Carter, C., Meier, W.: Temporally resolved planar measurements of transient phenomena in a partially pre-mixed swirl flame in a gas turbine model combustor. Combust. Flame 157, $1510-1525$ (2010)

62. Navarro-Martinez, S., Kronenburg, A.: Flame stabilization mechanisms in lifted flames. Flow Turbul. Combust. 87, 377-406 (2011)

63. Ayache, S., Mastorakos, E.: Investigation of the "TECFLAM" non-premixed flame using large eddy simulation and proper orthogonal decomposition. Flow Turbul. Combust. 90, 219-241 (2013)

64. Frank, J.H., Kaiser, S.A., Oefelain, J.C.: Analysis of scalar mixing dynamics in LES using highresolution imaging of laser Rayleigh scattering in turbulent non-reacting jets and non-premixed jet flames. Proc. Combust. Inst. 33, 1373-1381 (2011)

65. Pitsch, H., Steiner, H.: Scalar mixing and dissipation rate in large-eddy simulations of non-premixed turbulent combustion. Proc. Combust. Inst. 28, 41-49 (2000) 